\title{
Intermediate to low-mass stellar content of Westerlund $1^{\star}$
}

\author{
W. Brandner ${ }^{1,2}$, J. S. Clark ${ }^{3}$, A. Stolte ${ }^{2}$, R. Waters ${ }^{4}$, I. Negueruela ${ }^{5}$, and S. P. Goodwin ${ }^{6}$ \\ 1 Max-Planck-Institut für Astronomie, Königstuhl 17, 69117 Heidelberg, Germany \\ e-mail: brandner@mpia.de \\ 2 UCLA, Division of Astronomy, Los Angeles, CA 90095-1547, USA \\ 3 Open University, UK \\ ${ }^{4}$ Sterrenkundig Instituut "Anton Pannekoek", Amsterdam, The Netherlands \\ 5 Dpto. de Física, Ingeniería de Sistemas y Teoría de la Señal, Universidad de Alicante, 03080 Alicante, Spain \\ ${ }^{6}$ Dept. of Physics \& Astronomy, University of Sheffield, Sheffield, UK
}

Received 1 April 2007 / Accepted 31 October 2007

\section{ABSTRACT}

\begin{abstract}
We have analysed near-infrared NTT/SofI observations of the starburst cluster Westerlund 1, which is among the most massive young clusters in the Milky Way. A comparison of colour-magnitude diagrams with theoretical main-sequence and pre-main sequence evolutionary tracks yields improved extinction and distance estimates of $A_{K \mathrm{~s}}=1.13 \pm 0.03 \mathrm{mag}$ and $d=3.55 \pm 0.17 \mathrm{kpc}(D M=$ $12.75 \pm 0.10 \mathrm{mag}$ ). The pre-main sequence population is best fit by a Palla \& Stahler isochrone for an age of $3.2 \mathrm{Myr}$, while the main sequence population is in agreement with a cluster age of 3 to $5 \mathrm{Myr}$. An analysis of the structural parameters of the cluster yields that the half-mass radius of the cluster population increases towards lower mass, indicative of the presence of mass segregation. The cluster is clearly elongated with an eccentricity of 0.20 for stars with masses between 10 and $32 M_{\odot}$, and 0.15 for stars with masses in the range 3 to $10 M_{\odot}$. We derive the slope of the stellar mass function for stars with masses between 3.4 and $27 M_{\odot}$. In an annulus with radii between 0.75 and $1.5 \mathrm{pc}$ from the cluster centre, we obtain a slope of $\Gamma=-1.3$. Closer in, the mass function of Westerlund 1 is shallower with $\Gamma=-0.6$. The extrapolation of the mass function for stars with masses from 0.08 to $120 M_{\odot}$ yields an initial total stellar mass of $\approx 52000 M_{\odot}$, and a present-day mass of 20000 to $45000 M_{\odot}$ (about 10 times the stellar mass of the Orion nebula cluster, and 2 to 4 times the mass of the NGC 3603 young cluster), indicating that Westerlund 1 is the most massive starburst cluster identified to date in the Milky Way.
\end{abstract}

Key words. stars: evolution - stars: formation - stars: luminosity function, mass function - stars: pre-main sequence stars: supernovae: general - Galaxy: open clusters and associations: individual: Westerlund 1

\section{Introduction}

Clustered star formation is the dominant mode of star formation in the Universe with about $80 \%$ of all stars originating in giant molecular clouds (Lada \& Lada 2003). The globular cluster systems in the Milky Way or M31 might be remnants of an epoch of Super Star Cluster (SSC) formation early in the history of these galaxies. Low-mass stars lost in the course of the dynamical evolution of the SSCs could explain the majority of Pop II field stars found in galaxy halos (Kroupa \& Boily 2002). While the Galactic globular clusters formed more than 12 Gyr ago, 100s of young SSCs with masses comparable to or greater than Galactic globular clusters; i.e., present-day masses $\geq 100000 M_{\odot}$, have been identified in interacting galaxies like, e.g., the Antennae galaxies (Whitmore \& Schweizer 1995).

At extragalactic distances, however, individual cluster members are often unresolved, and physical properties of SSCs - such as their initial mass function (IMF) and total mass, degree of binarity, or density profile - must be estimated from their integrated spectra and photometry alone, which in turn are dominated by the most massive, luminous stars present. The contribution of lower mass stars to the total cluster mass, which is

* Based on observations collected at the European Southern Observatory, La Silla, Chile, and retrieved from the ESO archive (Prog ID 67.C-0514). crucial for the dynamical evolution and the long-term survival of a cluster (e.g., de Grijs \& Parmentier 2007, and references therein), remains uncharted. Indirect estimates of the total cluster mass can be derived by extrapolating the IMF towards lower masses. Another possibility is to determine the dynamical mass of a cluster from its half mass radius and velocity dispersion under the assumption of virial equilibrium. Studies by different groups either agreed with a Kroupa (2002) IMF over the whole range of stellar masses (e.g. Larsen \& Richtler 2004) or found a truncated IMF towards lower stellar masses (e.g., Rieke et al. 1993; McCrady et al. 2005). Young, massive clusters, however, are not necessarily in virial equilibrium, as photoionization and winds from O-stars in their centres rapidly expel any remnant gas left over from the formation process (Kroupa \& Boily 2002; Bastian \& Goodwin 2006).

The determination of the IMF slope as well as the potential presence or absence of a low mass stellar component, is critical for constraining the long term evolution of such clusters. This addresses the question of whether (proto-) Globular Clusters are still forming in the Universe today. Moreover, as the majority of low mass field stars might have formed in clusters, the uncertainty in the initial ratio of high to low mass stars in clusters results in a corresponding uncertainty in the star formation and chemical enrichment history of galaxies like our Milky Way.

Therefore we examine starburst clusters in the Milky Way and its companion galaxies, where SSCs can be resolved into 
individual stars, and cluster properties like the IMF can be determined directly. As a starburst cluster we consider any cluster which is massive enough to house several early O-type stars with initial masses in excess of $50 M_{\odot}$ in its centre. Among the most massive Galactic starburst clusters studied to date are NGC 3603YC (e.g., Brandl et al. 1999; Stolte et al. 2006) and Westerlund 2 (Wd 2, Ascenso et al. 2007), both in the Carina spiral arm, and the two massive clusters Arches (Stolte et al. 2002, 2005; Figer et al. 2002; Kim et al. 2006) and Quintuplet (e.g., Cotera et al. 1996; Figer et al. 2004) in the Galactic centre (GC) region.

One questions of particular interest is whether or not the presence of high mass stars hinders or even quenches the formation of low mass stars. Intense UV radiation fields and strong stellar winds of nearby OB stars might lead to the destruction of protostellar cores before low-mass stars can form. Furthermore, in extreme environments like the GC region, variations in the physical conditions leading to higher thermal and/or turbulent pressure, as well as the presence of strong magnetic fields might preferentially yield higher mass prestellar cores than in more benign environs (Morris 1993; Stolte et al. 2005; Larson 2006; Klessen et al. 2006).

Along with the Arches and Quintuplet clusters in the GC region, and the NGC 3603YC in the Sagittarius-Carina spiral arm, Westerlund 1 (Wd 1), located in the Scutum-Crux spiral arm, is one of the very few young, massive starburst clusters in the Milky Way.

Discovered by Westerlund (1961), subsequent optical observations of the galactic open cluster Westerlund 1 (Wd 1) suggested an unusually rich population of cool and hot supergiants (Westerlund 1987), from which a large cluster mass could be inferred. Recent optical spectroscopic and photometric observations have confirmed these assertions (Clark \& Negueruela 2002; Clark et al. 2005 (henceforth C05); Negueruela \& Clark 2005). Located close to the Galactic plane $\left(b=-0.35^{\circ}\right)$ at a distance of $\sim 3-5 \mathrm{kpc}, \mathrm{Wd} 1$ is subject to significant foreground extinction. Thus optical studies of the cluster were limited to the most massive stars of $\mathrm{Wd1}$. C05 spectroscopically identified about 50 cluster members with masses in excess of $30 M_{\odot}$. Adding 150 probable cluster members identified by means of photometry, they estimate that the high-mass stellar content of Wd 1 alone amounts to $6000 M_{\odot}$. Assuming a Kroupa IMF to extrapolate from this population, $\mathrm{C} 05$ then infer a likely total mass of $\sim 10^{5} M_{\odot}$ for Wd1; directly comparable to the masses of SSCs observed in other galaxies.

Up to now, however, only the evolved, high-mass stellar population of the cluster has been characterised. The presence of Wolf-Rayet stars (e.g., Crowther et al. 2006; Skinner et al. 2006a), a pulsar indicative of a recent supernova (Skinner et al. 2006b; Muno et al. 2006a), as well as O supergiants, suggest an age between 3 and $5 \mathrm{Myr}$ for the cluster. Because of a visual foreground extinction of $A_{V} \approx 10 \mathrm{mag}$, and the uncertainties in the intrinsic luminosity of the evolved, massive stars, distance determinations to $\mathrm{Wd} 1$ range from 2 to $5.5 \mathrm{kpc}(\mathrm{C} 05)$.

Because of its proximity, Wd1 provides the unique opportunity to directly determine the spatially resolved IMF of a SSC and, given that it is located well away from the GC in a supposedly 'quiescent' region of the Galactic disc, potentially study physical properties imprinted by the formation process from the parental molecular cloud on the cluster. With the aim to better determine basic cluster properties such as age, foreground extinction and distance, to directly measure the IMF, and to study the structural properties of $\mathrm{Wd} \mathrm{1,} \mathrm{we} \mathrm{have} \mathrm{analysed} \mathrm{deep} \mathrm{near}$ IR observations. This paper describes the analysis of NTT/SofI
Table 1. Observing log for the NIR data. The observations were carried out with the ESO NTT and SofI on 9 June 2001.

\begin{tabular}{lcccr}
\hline \hline Object & Filter & $\begin{array}{c}t_{\text {int }} \\
{[\mathrm{s}]}\end{array}$ & $\begin{array}{c}\text { seeing } \\
{\left[{ }^{\prime \prime}\right]}\end{array}$ & $\begin{array}{r}\text { sat. limit } \\
{[\mathrm{mag}]}\end{array}$ \\
\hline Westerlund 1 & $\mathrm{J}$ & 120 & 0.81 & 10.1 \\
Westerlund 1 & $\mathrm{H}$ & 120 & 0.82 & 9.5 \\
Westerlund 1 & $\mathrm{Ks}$ & 120 & 0.73 & 9.1 \\
Off-Field & $\mathrm{J}$ & 120 & 0.77 & 10.2 \\
Off-Field & $\mathrm{H}$ & 120 & 0.77 & 9.6 \\
Off-Field & $K \mathrm{~s}$ & 120 & 0.74 & 9.1 \\
\hline
\end{tabular}

observations of $\mathrm{Wd} 1$ covering a $\sim 5^{\prime} \times 5^{\prime}$ field of view centred on the cluster core, which are sensitive to $\sim$ solar mass stars at a distance of $4 \mathrm{kpc}$; a second paper details subsequent VLT/NAOSCONICA adaptive optics observations of selected core regions sensitive to $\sim 0.2 M_{\odot}$.

The structure of the paper is as follows: in Sect. 2 we present the observations and the data analysis. The basic astrophysical quantities extinction, distance, and age of $\mathrm{Wd} 1$ are derived in Sect. 3. Section 4 discusses the mass function and the total stellar mass of Wd 1. Dynamical and structural parameters of Wd 1 are addressed in Sect. 5, followed by a comparison of Wd 1 to other well studied starburst clusters in Sect. 6. Section 7 summarises the results.

\section{Observations and data reduction}

\subsection{NTT/Sofl observation}

JHKs-broad band imaging observations of $\mathrm{Wd} 1$ (centred on $\left.\operatorname{RA}(2000)=16^{\mathrm{h}} 47^{\mathrm{m}} 03^{\mathrm{s}}, \operatorname{Dec}(2000)=-45^{\circ} 50^{\prime} 37^{\prime \prime}\right)$ and a nearby comparison field (located $\approx 7^{\prime}$ to the east, and $\approx 13^{\prime}$ to the south of Wd 1 , and centred on $\operatorname{RA}(2000)=16^{\mathrm{h}} 47^{\mathrm{m}} 43^{\mathrm{s}}, \operatorname{Dec}(2000)=$ $-46^{\circ} 03^{\prime} 47^{\prime \prime}$ ), each covering an area of $4.5^{\prime} \times 4.5^{\prime}$ (corresponding to $4.8 \mathrm{pc} \times 4.8 \mathrm{pc}$ at the distance of $\mathrm{Wd} 1)$, obtained with NTT/SofI (PI: J. Alves) were retrieved from the ESO archive (see Fig. 1). SofI is equipped with a Hawaii $\mathrm{HgCdTe}$ detector. The observations were obtained with a plate scale of $0.29^{\prime \prime} /$ pixel. Individual integration times were $1.2 \mathrm{~s}$ (DIT) and 10 frames were co-added, resulting in $12 \mathrm{~s}$ of integration time per dither position. For each field and filter a total of 10 dither positions were used, resulting in a total integration time of $120 \mathrm{~s}$ in each filter.

\subsection{Eclipse raw data reduction}

Data reduction was carried out using the eclipse jitter routines (Devillard 2001). Because of the high degree of crowding in the cluster field, sky frames derived from the comparison field were used in the reduction of the $\mathrm{Wd} 1$ frames. The resolution on the final images is $\approx 0.75^{\prime \prime}$ to $0.80^{\prime \prime}$ (see Table 1 for more details).

\subsection{Photometric analysis and calibration}

PSF fitting photometry in $J, H$, and $K$ s for 7000 stars in the Wd 1 area, and 5300 stars in the off-field was derived using the IRAF implementation of DAOPHOT (Stetson 1987). Photometric zeropoints and colour terms were computed by comparison of instrumental magnitudes of relatively isolated, bright sources with photometry from the 2MASS point source catalogue.

The resulting $K \mathrm{~s}$ vs. $J-K \mathrm{~s}$ colour-magnitude diagrams (CMD) for Wd1 and the off-field for all stars with DAOPHOT fitting errors $\leq 0.2 \mathrm{mag}$ are presented in Fig. 2. The dashed line 

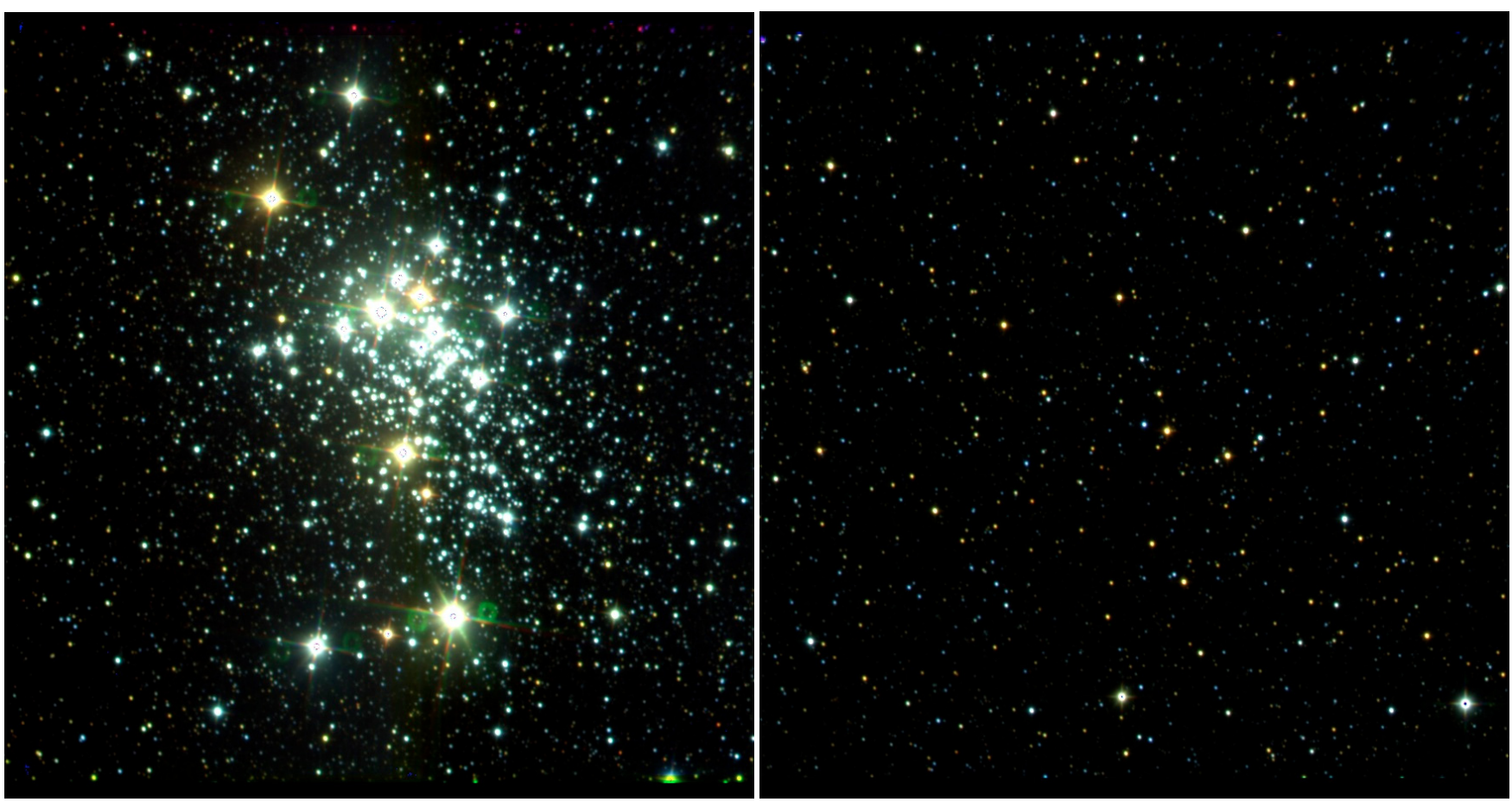

Fig. 1. Composite colour $J H K$ s images of Westerlund 1 (left $)$ and the comparison field (right) obtained with NTT/SofI. The field of view is $4^{\prime} \times 4^{\prime}$. North is up, and east is to the left.
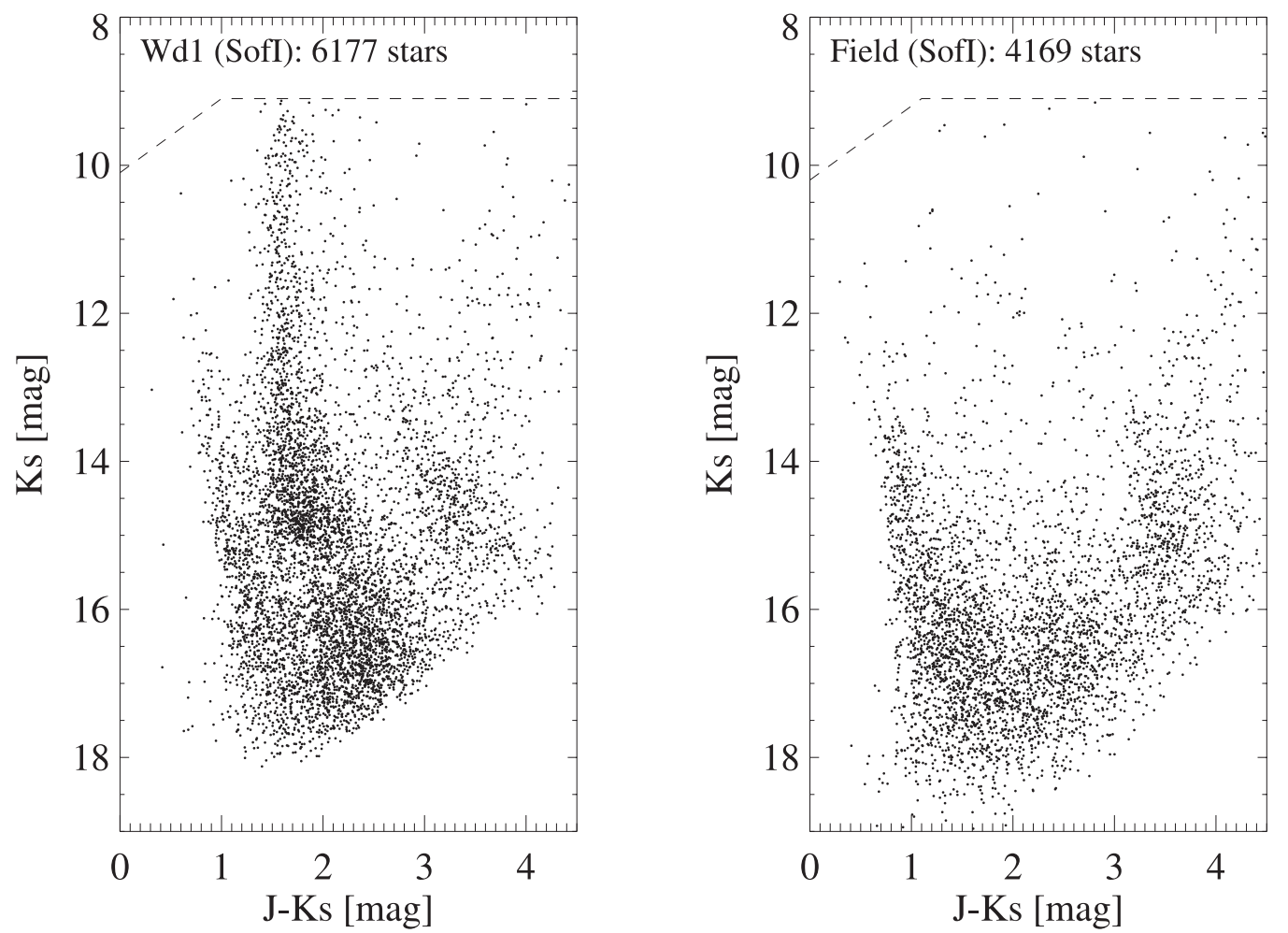

Fig. 2. Colour magnitude diagram of the cluster (including field contamination, left), and the comparison field (right). The dashed line marks the saturation limit.

indicates the saturation limit (see Table 1). The off-field is characterised by a blue main-sequence of foreground stars, and a red sequence of background stars, most of which could be red giants located in the galactic bulge. The Wd 1 field shows the main-sequence and pre-main sequence members of the cluster in addition to the two populations visible in the off-field. Note that the red background population appears to be less reddened close to Wd 1 compared to the off-field. This is most likely due to varying extinction along the line of sight towards the bulge at distances larger than the distance to $\mathrm{Wd} 1$.

In the following analysis, only stars with DAOPHOT photometric fitting errors of 0.2 mag or less are considered. 

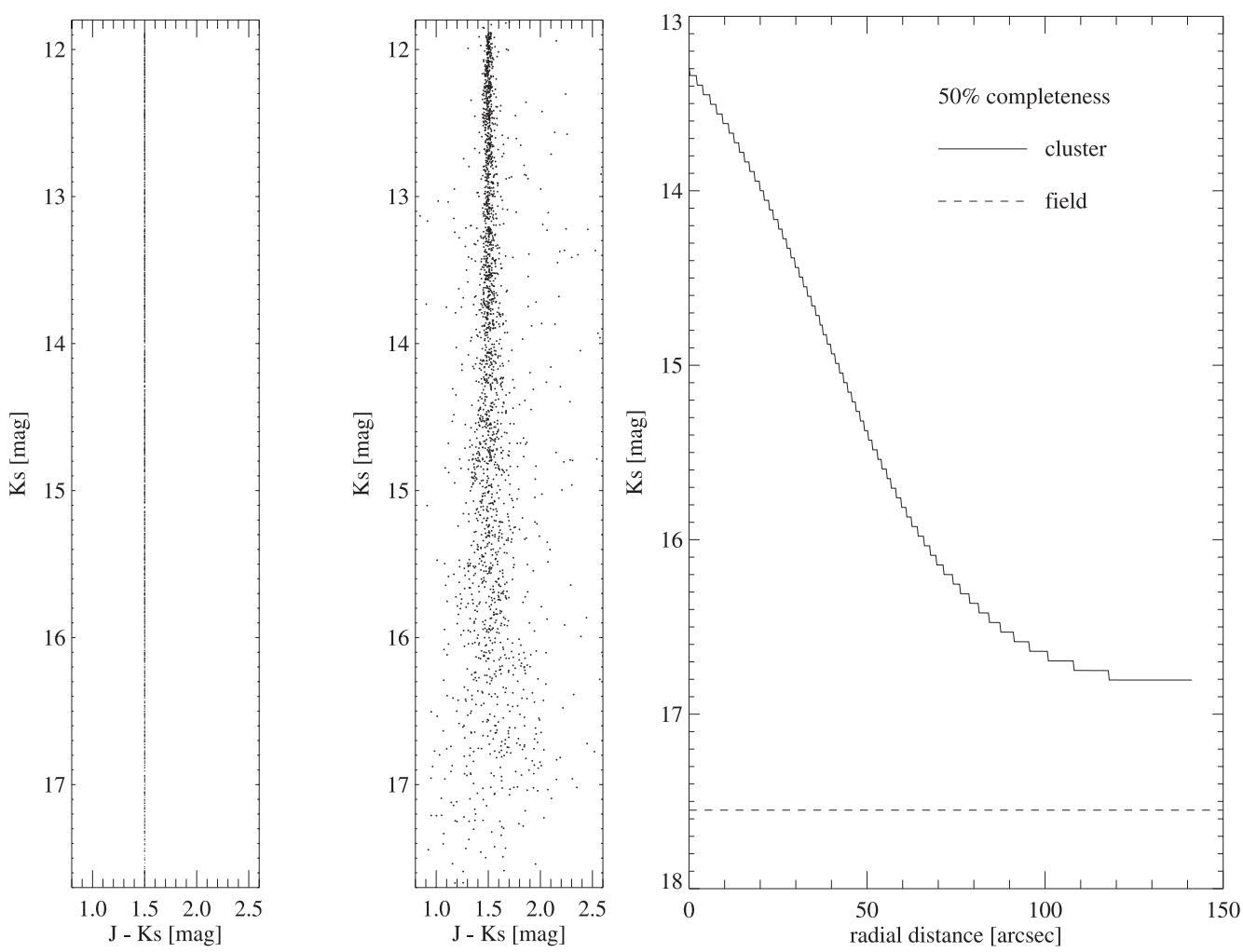

Fig. 3. Results of the completeness simulations for Westerlund 1. The two figures on the left show the input magnitudes and colours and the recovered magnitudes and colours, respectively, for the cluster frame. From this we also derive the photometric uncertainties. The figure on the right shows the $50 \%$ completeness limits. For the cluster, increasing crowding towards its center pushes the completeness limit (solid line) towards brighter magnitudes. The homogeneous stellar density in the comparison field results in an overall $50 \%$ completeness level down to $\mathrm{Ks}=17.55 \mathrm{mag}$ (dashed line).

\subsection{Incompleteness characterisation}

Incompleteness simulations with the aim to assess the effect of crowding on our ability to detect faint sources were carried out both for the jitter combined off-field and the cluster field. In order not to change the crowding characteristics of the frames, for each run only 50 stars were added using addstars under DAOPHOT. Artificial star magnitudes were allowed to scatter \pm 0.5 mag around a predefined value, and the stars were placed at random positions on the $K$ s-band frames. The detection experiment was following the same steps and using the same PSF as the initial DAOPHOT analysis. Only those stars, whose recovered magnitudes match to within \pm 0.5 mag the input magnitude, were counted in the final analysis. For each predefined magnitude, 10 such frames were created and analysed, i.e., $10 \times 50$ stars $=500 \mathrm{stars} / \mathrm{mag}$ bin. Then the magnitude was increased by $1.0 \mathrm{mag}$, and 10 new frames were created and analysed. This procedure was repeated $6 \times$, probing the magnitude range $K \mathrm{~s}=11.9 \mathrm{mag}$ to $17.9 \mathrm{mag}$ for the cluster frame, and $K \mathrm{~s}=13.9$ to $19.9 \mathrm{mag}$ for the field frame. For the $J$-band incompleteness simulations, stars were placed at the same positions as on the $K$ s-band frames. $J$-band magnitudes were computed assuming $J-K \mathrm{~s}=2.0 \mathrm{mag}$, which is an intermediate colour between the (lower) main-sequence stars in Westerlund 1 with $J-K \mathrm{~s} \approx 1.5 \mathrm{mag}$, and the pre-main sequence stars with $J-K \mathrm{~s} \approx$ $2.3 \mathrm{mag}$.

In total 240 frames with artificial stars added were analysed. The results are summarised in Fig. 3. Note that the lack of bright stars in the off-field pushes the $50 \%$ completeness limit to fainter magnitudes compared to the cluster field.

\subsection{Statistical field subtraction}

With Galactic coordinates $l \approx 339.55^{\circ}$ and $b \approx-0.40^{\circ}$, Wd 1 is embedded in a rich population of fore- and background stars. The more than 4000 stars detected in $J$ and $K$ s in the comparison field with DAOPHOT fitting errors $\leq 0.2$ mag (Fig. 2, right) give a good estimate of the field star population mix. This provides a firm basis to remove the field star contamination in the cluster $\mathrm{CMD}$, and hence determine a clean, relatively unbiased cluster population for further analysis.

The statistical field subtraction is based on a comparison of the cluster and the field CMD. The CMDs are subdivided into grid cells with a step size of 0.5 mag in colour and magnitude. The number of field stars within each cell is counted, normalised to the ratio of the sky areas covered by the image and the selected cluster annuli, and corrected for the differences in completeness fraction between the field and the cluster (see Fig. 3). Finally, the same number of stars is subtracted at random from the corresponding grid cell in the cluster CMD. Examples of the cleanedup ("field subtracted") cluster CMDs are shown in Fig. 4.

\section{Extinction, cluster age, distance and evolutionary phases of cluster members}

A starburst cluster most likely forms in a single event ("burst"), resulting in a small age spread of its stellar population. Galactic starburst clusters like NGC 3603 or Arches with several 1000 to 10000 stars spanning a factor of 100 or more in individual stellar mass have the advantage that global properties like foreground extinction, distance or age can be derived with a relatively small uncertainty by comparison to theoretical isochrones. For a 

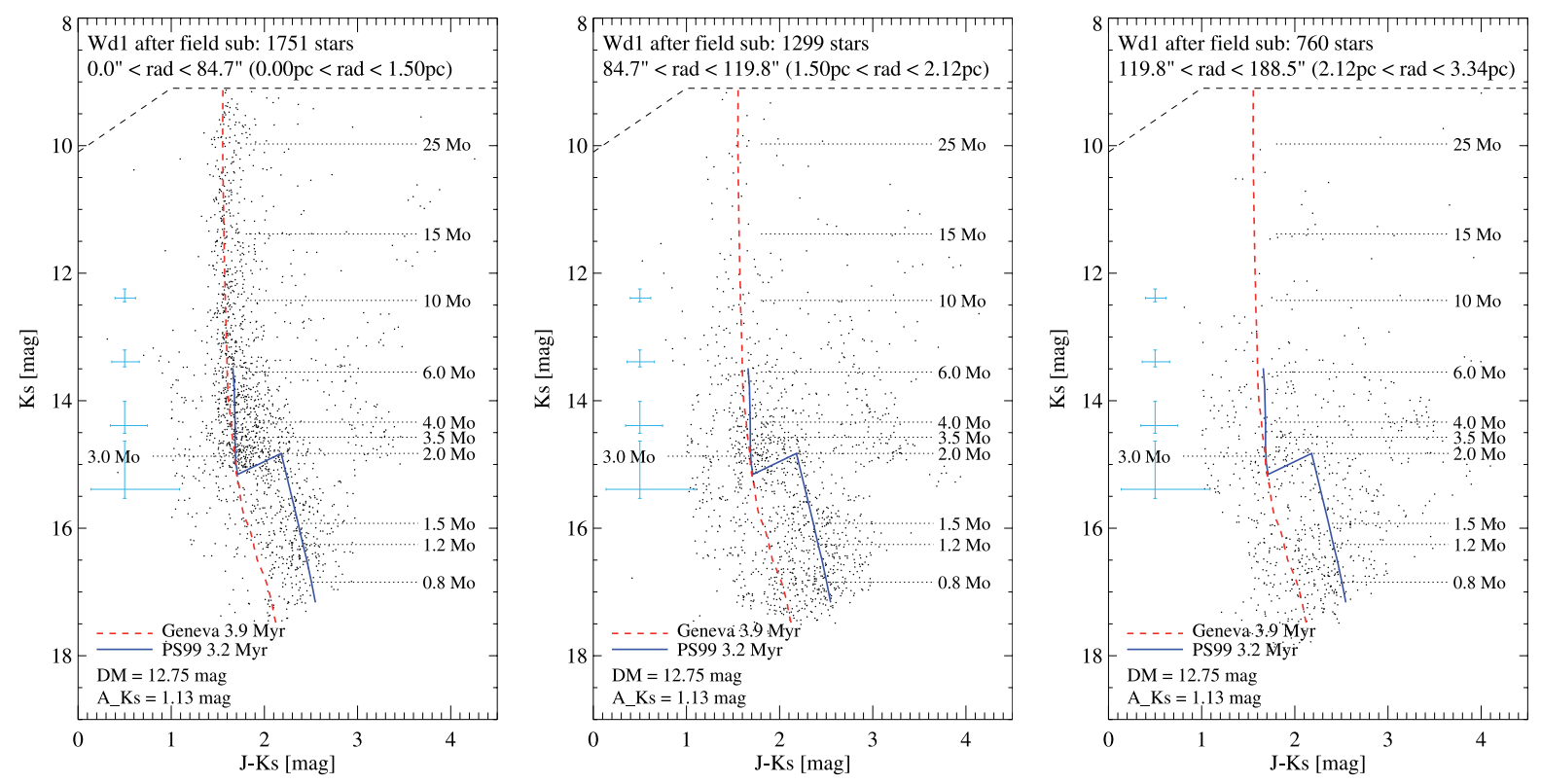

Fig. 4. Field subtracted CMDs of the cluster for 3 annuli. A 3.9 Myr Geneva ( $Z=0.020$, with overshooting and OPAL opacities) and a 3.2 Myr Palla \& Stahler isochrone are overplotted for comparison. The dashed line marks the saturation limit. Photometric uncertainties in $K$-magnitude and $J-K$ colour as determined by the artificial star tests to determine incompleteness are plotted to the left of the cluster sequence. The dotted lines indicate stellar masses of cluster members according to the Geneva isochrone for masses $\geq 6.0 M_{\odot}$, and the Palla \& Stahler isochrone for masses less than $6.0 M_{\odot}$.

detailed analysis of the mass function and the dynamical properties of $\mathrm{Wd} 1$, these basic astrophysical quantities have to be derived first.

For the following analysis we use Geneva isochrones (Lejeune \& Schaerer 2001) describing main-sequence and postmain sequence evolution of stars with masses between 0.8 and $120 M_{\odot}$, and Palla \& Stahler isochrones (Palla \& Stahler 1999) describing pre-main sequence and main-sequence evolution of stars with masses between 0.1 and $6 M_{\odot}$. In the following paper describing the analysis of the adaptive optics data, we will provide a more in-depth comparison of evolutionary tracks. Isochrones by Palla \& Stahler are preferred over other, more recent tracks (e.g., Siess et al. 2000) as they provide a better fit to the pre-main sequence/main sequence transition region in near infrared CMDs of starburst clusters. In their comparison of NIR observations of NGC 3603 YC with PS99 and Yonsei-Yale (Yi et al. 2001) isochrones, Stolte et al. (2005) also found a better fit of the PS99 tracks to the observed pre-main sequence/main sequence transition region. Starburst clusters, i.e. clusters housing early O-type stars in their centres, might be special in this respect, as the intense UV radiation of the most massive cluster members rapidly photo-evaporates any remaining accretion disks or envelopes around lower mass stars in the cluster (e.g., Brandner et al. 2000 and references therein). Thus pre-main sequence evolution in starburst clusters seems to be well described by non-accreting tracks. For a thorough discussion of shortcomings of theoretical isochrones compared to observations we refer to Mayne et al. (2007). Using the same set of isochrones as previously used for the analysis of the mass functions of NGC 3603 and Arches also facilitates a direct comparison of the 3 clusters. We use a mass of $6.0 M_{\odot}$ as the transition point from Geneva to Palla \& Stahler isochrones as both sets of tracks predict the same NIR magnitudes for stars of this mass in the age range considered.

The values for extinction, distance modulus and age of the cluster, which we present in the following, were derived in an iterative process by fitting isochrones to the SofI and NACO near-infrared data.

\subsection{Extinction}

As the intrinsic $J-K$ s colours of main sequence stars with masses in the range 6 to $30 M_{\odot}$ just vary between -0.1 and $-0.2 \mathrm{mag}$, the foreground extinction can be derived by simply fitting a zeroage main-sequence (ZAMS) to the main-sequence population of Wd 1 and assuming a standard Rieke \& Lebofsky (1985) extinction law. In Fig. 5 only stars in the SofI data set with DAOPHOT fitting errors less equal $0.05 \mathrm{mag}$ are shown. The artificial star tests carried out as part of the incompleteness simulation indicate that the DAOPHOT fitting errors underestimate the true photometric errors. Interestingly, the recovered magnitudes are on average brighter than the input magnitudes of the artificial stars. This asymmetry in the photometric errors around zero is also present in the colour estimate $J-K \mathrm{~s}$, though less pronounced. The photometric errors explain part of the observed scatter in the colour of the main sequence stars. By comparing CMDs for different regions in our field of view, we also see evidence for differential extinction. In general, the regions to the west and south of the cluster centre suffer slightly lower foreground extinction than the regions to the east and north of the cluster centre. As a best fit, we get $A_{K \mathrm{~s}}=1.13 \pm 0.03 \mathrm{mag}$. Since all our measurements are in the near-infrared, and the theoretical isochrones used have been transformed to JHK-magnitudes and colours, the results are relatively insensitive to any deviation from a standard extinction law.

In their analysis of the NIR colours of $18 \mathrm{~W}-\mathrm{R}$ stars in Wd 1 Crowther et al. (2006) derived $A_{\mathrm{Ks}}=0.96 \pm 0.14 \mathrm{mag}$, which within the quoted uncertainties overlaps with the $K \mathrm{~s}$-band extinction derived by us. We note, however, that for the two W-R stars in overlap with our sample (see Table 4), Crowther et al. (2006) observe $J-K$ s colours 0.35 to 0.46 mag bluer than we do. Variability as reported by Bonanos (2007) might explain part of 


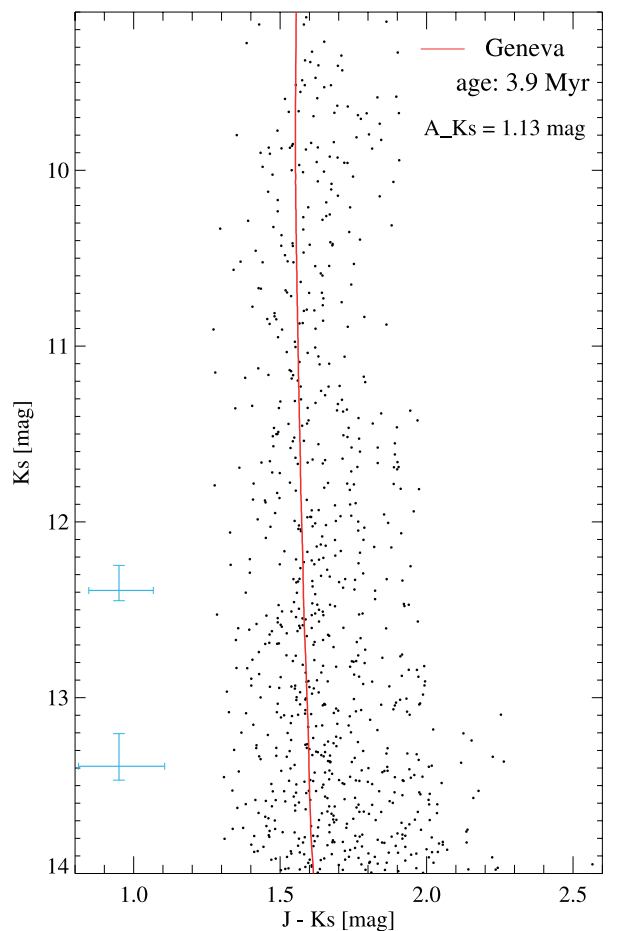

Fig. 5. Colour magnitude diagram of the main sequence population of the cluster for stars with DAOPHOT fitting uncertainties smaller than $0.05 \mathrm{mag}$ in $\mathrm{J}$ and Ks. A Geneva isochrone (solid line, age of 3.9 Myr, $D M=12.75 \mathrm{mag}$ ) for $A_{\mathrm{K}}=1.13 \mathrm{mag}$ is overplotted. Photometric uncertainties (root mean square) as determined by the artificial star test are indicated to the left of the cluster main sequence. Even though some of the stars with colours 0.3 to 0.5 mag redder than the main-sequence have been identified as W-R stars, the relatively wide colour range is in part due to differential foreground reddening.

this discrepancy, though there might also be a systematic offset in the zeropoint calibrations of the two data sets.

For comparison to other extinction determinations, which were exclusively based on studies in the visual, we also compute the visual extinction. Assuming a normal extinction law with $R=A_{V} / E_{B-V}=3.1, A_{K \mathrm{~s}}=1.13 \mathrm{mag}$ corresponds to $A_{V} \approx 10.1$ mag (Rieke \& Lebofsky 1985).

Westerlund (1961) derived an optical colour $V-I=$ +4.5 mag for the $\approx 80$ brightest stars of the cluster. By assuming an intrinsic colour of $(V-I)_{0}=-0.35$, which is typical for early type stars, this corresponds to $E(V-I)=4.85 \mathrm{mag}$. If one further assumes a standard extinction law with $A_{V}=1.93 E(V-I)$ (see Rieke \& Lebofsky 1985), this results in $A_{V} \approx 9.4 \mathrm{mag}$. Note that Westerlund (1961) deduced a higher $A_{V}$ of $11.2-12 \mathrm{mag}$ by assuming a ratio between total and selective absorption of $2.3 \pm 1.4$ (Kron \& Mayall 1960). Follow-up photometric and spectroscopic observations considering about 260 cluster members yielded $A_{V} \approx 9.7 \pm 0.8 \mathrm{mag}$ (Westerlund 1987). Clark et al. (2005) estimate $A_{V}=11.6$ to $13.6 \mathrm{mag}$, and present evidence for anomalous extinction towards $\approx 20$ OB supergiants in $\mathrm{Wd} 1$. While for normal interstellar extinction the ratio of total to selective extinction $R=3.1, R$ values in the range 1.7 to 5.5 have been observed in high-mass star forming regions (Patriarchi et al. 2001, but see also Hadfield \& Crowther 2006 and Lamzin 2006). For $R=3.7, A_{K}=1.13 \pm 0.03 \mathrm{mag}$ corresponds to $A_{V} \approx 12.0 \mathrm{mag}$, i.e. in the range of the visual extinction values determined by Clark et al. (2005) towards some of the evolved stars in $\mathrm{Wd} 1$.

\subsection{Age}

For the age determination, we take advantage of the fact that the width and the shape of the pre-main sequence - main-sequence transition region in $J-K \mathrm{~s}$ varies with age. This allows us to derive the age of the cluster by comparison with theoretical isochrones. The best fitting Palla \& Stahler isochrone are for ages of 3.2 to $3.4 \mathrm{Myr}$. Isochrones for an age of $3.0 \mathrm{Myr}$ or 3.6 Myr, respectively do not fit as well, in particular when taking the adaptive optics data into account. A more detailed comparison of different sets of theoretical isochrones will be presented in the second paper, which features a detailed analysis of the cluster population in the range of 0.4 to $3 M_{\odot}$. We estimate the age of the low-mass stellar population of $\mathrm{Wd} 1$ since crossing the "birthline" (see Stahler 1983) to $3.3 \pm 0.2 \mathrm{Myr}$.

At young ages of $\leq 10 \mathrm{Myr}$, pre-main sequence evolutionary tracks originating at the stellar birthline, and mainsequence/post-main-sequence evolutionary tracks originating at the zero-age-main-sequence are not necessarily on the same absolute timescale, and might show systematic offsets. This is exemplified by the Orion nebula cluster (ONC), for which Meynet et al. (1993) fit the youngest Geneva isochrone in their grid for an age of $4 \mathrm{Myr}$ to the upper main sequence of ONC, while the canonical age for the low mass stars is $<2 \mathrm{Myr}$ (Hillenbrand 1997; Palla \& Stahler 1999).

In the mass and wavelength (colour) range accessible with the SofI near-infrared data, Geneva isochrones (Lejeune \& Schaerer 2001) are rather insensitive to age, in particular as nearinfrared colours like $J-K$ s always sample the Rayleigh-Jeans tail in the spectra of the hot, massive stars. Consequently, we cannot use the Geneva isochrones to derive further constraints on the age of $\mathrm{Wd} \mathrm{1.} \mathrm{Based} \mathrm{on} \mathrm{the} \mathrm{observations} \mathrm{of} \mathrm{evolved} \mathrm{stars} \mathrm{in} \mathrm{Wd} 1$, both Clark et al. (2005) and Crowther et al. (2006) argue for an age of 4 to $5 \mathrm{Myr}$. In the following analysis, we use Geneva isochrones in the age range 3.0 to 5.0 Myr. The isochrones are based on the "classical" evolutionary tracks including overshoot, and have been computed for a metallicity of $Z=0.02$.

As an average age for the low $(3.3 \pm 0.2 \mathrm{Myr})$ and high-mass $(3.9 \pm 1.0 \mathrm{Myr})$ stellar population of $\mathrm{Wd} 1$, we adopt $t_{\text {cluster }}=$ $3.6 \pm 0.7 \mathrm{Myr}$. This also results in consistent values for the brightness and colours predicted by both sets of tracks for the $6 M_{\odot}$ stars.

\subsection{Distance}

Once extinction and age have been established, the distance to the cluster follows straight from the observed near-infrared magnitude of the transition region between pre-main sequence and main-sequence. For the 3.2 Myr PS99 isochrone, we get $D M=12.75 \pm 0.10 \mathrm{mag}$ or $d=3.55 \pm 0.17 \mathrm{kpc}$.

This is in agreement with the study by Westerlund (1987), who derived a distance modulus of $D M=13.6 \pm 0.7 \mathrm{mag}$, as well as Clark et al. (2005), who argue for a distance between 2 and $5.5 \mathrm{kpc}$, and Crowther et al. (2006), who derive $D M=$ $13.50 \pm 0.66$ mag based on the analysis of the NIR colours of 18 W-R stars in Wd 1. More recently, Kothes \& Dougherty (2007) derived a distance of $3.9 \pm 0.7 \mathrm{kpc}$ based on the radial velocity of an HI feature likely associated with $\mathrm{Wd} 1$, assuming a standard Galactic rotation curve and a distance of $7.6 \mathrm{kpc}$ (Eisenhauer et al. 2005; Reid 1993) between the Sun and the GC.

The results on extinction, age and distance are summarised in Table 2. 
Table 2. Basic astrophysical parameters extinction, $D M$ (distance), metallicity and age of $\mathrm{Wd} 1$ as derived from comparison of the $K s$ vs. $J-K$ s CMD with theoretical Geneva and Palla \& Stahler isochrones.

\begin{tabular}{cccc}
\hline \hline $\begin{array}{c}A_{K s} \\
\mathrm{mag}\end{array}$ & $\begin{array}{c}D M \\
{[\mathrm{mag}]}\end{array}$ & $Z$ & $\begin{array}{c}\text { age } \\
\mathrm{Myr}\end{array}$ \\
\hline $1.13 \pm 0.03$ & $12.75 \pm 0.10$ & 0.02 & $3.6 \pm 0.7$ \\
& $(3.55 \pm 0.17 \mathrm{kpc})$ & & \\
\hline
\end{tabular}

Table 3. Mass function slope $\Gamma$ for stars with masses between 3.4 and $27 M_{\odot}$.

\begin{tabular}{c|ccc|c}
\hline \hline Radial Dist. & $3.2 \mathrm{Myr}$ & $3.9 \mathrm{Myr}$ & $4.9 \mathrm{Myr}$ & $3.9 \mathrm{Myr}$ \\
& $z=0.02$ & $z=0.02$ & $z=0.02$ & $z=0.04$ \\
\hline $0-0.75 \mathrm{pc}$ & -0.7 & -0.6 & -0.5 & -0.6 \\
$0.75-1.5 \mathrm{pc}$ & -1.4 & -1.3 & -1.3 & -1.4 \\
$1.5-2.1 \mathrm{pc}$ & -1.7 & -1.6 & -1.5 & -1.6 \\
$2.1-3.3 \mathrm{pc}$ & -1.7 & -1.7 & -1.9 & -2.0 \\
\hline
\end{tabular}

$\Gamma$ values are quoted for different annuli, and three Geneva isochrones for $z=0.020$ and for ages of 3.2, 3.9, and 4.9 Myr, respectively. The column to the right lists $\Gamma$ based on a Geneva isochrone with $z=0.040$ and for an age of 3.9 Myr. There is a slight dependence of $\Gamma$ on the age and the metallicity of the adopted isochrone.

\subsection{Evolutionary phase of cluster members}

Wd 1 provides a unique, and quite interesting snapshot of stellar evolution as a function of mass. At an age of $\approx 3.6 \mathrm{Myr}$, the most massive cluster members have already left the main-sequence. Only stars with $K \mathrm{~s} \geq 11 \mathrm{mag}$, corresponding to an MK-type of B0.5V $\left(m \approx 15 M_{\odot}\right)$, are still close to the ZAMS, whereas more massive stars already started to evolve off the ZAMS. The cluster main-sequence extends down to $K \mathrm{~s} \approx 15 \mathrm{mag}$, corresponding to an MK-type of A0V $\left(m=3 M_{\odot}\right)$. Stars with masses between 2 and $3 M_{\odot}$ are located in the pre-main sequence - main-sequence transition region, and stars with masses less than $2 M_{\odot}$ are still in the pre-main sequence phase.

Massive young clusters like Wd 1 offer the rare opportunity to bring evolutionary tracks for higher mass stars, starting with the zero-age main sequence, and pre-main sequence evolutionary tracks starting, e.g., with the stellar birthline (Stahler 1983) on the same evolutionary time scale. This is a crucial empirical calibration point in developing a consistent theory of stellar evolution encompassing all stars from $0.08 M_{\odot}$ to $120 M_{\odot}$.

\section{Mass function and cluster mass}

We compute the centre of $\mathrm{Wd} 1$ from the field-subtracted spatial distribution of cluster members. We find the cluster centre to be located at $\operatorname{RA}(2000)=16^{\mathrm{h}} 47^{\mathrm{m}} 04.0^{\mathrm{s}}$, Dec $(2000)=$ $-45^{\circ} 51^{\prime} 04.9^{\prime \prime}$. This location is used as a reference for the following discussion.

\subsection{Mass function and number of cluster members}

Individual masses for cluster members are derived by comparison of their location in the CMD with a 3.9 Myr Geneva isochrone for stars more massive than $6.0 \mathrm{M}_{\odot}$, and a $3.2 \mathrm{Myr}$ PS99 isochrone for stars with masses of $6.0 M_{\odot}$ and less. We note that in the evolutionary time scale defined by the tracks by Palla \& Stahler, a star with a mass of $3.5 M_{\odot}$ takes about $1.4 \mathrm{Myr}$ to cross from the birthline to the zero-age main sequence (ZAMS), while the location of the birthline for a star with $6.0 M_{\odot}$ coincides with the ZAMS. No attempt was made to correct for intrinsic infrared excess and/or extinction towards individual stars. This is justified by the fact that a $J-H$ vs. $H-K$ s colourcolour diagram of the cluster members indicates that only a small fraction (less than 10\%) have an intrinsic infrared excess. Stellar masses were assigned based on the JHKs-brightness and -colours. For stars above $3.5 M_{\odot}$, only those with $J-K$ s colours within -0.3 and +0.4 mag of the isochrone were considered for the mass function. As the pre-main sequence - main-sequence transition region marks an area with a broader spread in colour, for stars below $3.5 M_{\odot}$, the $J-K$ s colour range was extended to allow a variation of -0.3 to +0.6 mag around the colour indicated by the PS99 isochrone.

At an age of $3.9 \mathrm{Myr}$, a star with an initial mass of $30 M_{\odot}$ has lost about $3 \%$ or $6.5 \%$ of its initial mass for a metallicity of $Z=$ 0.02 or 0.04 , respectively, according to the Geneva evolutionary models. While this has a minute effect on the mass function, we assign initial rather than present-day masses to each of the stars. The mass function obtained this way is then corrected for incompleteness, and the slope of the mass function is computed for the range 3.4 to $27 M_{\odot}$.

Mass functions are traditionally visualised as histograms. Deriving the mass function slope from a histogram can be problematic, as the value of the fitted slope in general varies with bin size and locations (Maíz-Apellániz \& Úbeda 2005). Slopes derived from cumulative mass functions are much more robust (e.g., Stolte et al. 2006). In the following we discuss both standard histogram mass functions and cumulative mass functions.

Figure 6 shows the mass function of the cluster assuming solar metallicities for four annuli. The mass function slope $\Gamma$ has been fitted for the mass range 3.4 to $27 M_{\odot}$. With $\Gamma=-0.6$, the mass function is shallow in the inner $\approx 0.75 \mathrm{pc}$ with respect to a standard Salpeter mass function with $\Gamma=-1.35$, getting steeper for radial distances from the cluster centre between 0.75 and $1.5 \mathrm{pc}$ with $\Gamma=-1.3, \Gamma=-1.6$ for radii between $1.5 \mathrm{pc}$ and $2.1 \mathrm{pc}$, and $\Gamma=-1.7$ for radii larger than $2.1 \mathrm{pc}$. Because of the small number of high mass stars, as well as uncertainties in the statistical subtraction of field stars, the slopes in the two outermost annuli have uncertainties of $5 \%$ to $10 \%$, whereas the slopes in the inner annuli have uncertainties smaller than $5 \%$.

Table 3 summarises the derived mass function slopes for masses between 3.4 and $27 M_{\odot}$. The lower limit of $3.4 M_{\odot}$ ensures that also in the innermost annulus only stars with masses above the $50 \%$ completeness limit are included. The upper limit has been selected such that stars with $27 M_{\odot}$ are still below our photometric saturation limit even for an age of $4.9 \mathrm{Myr}$.

The cumulative mass function for the same four annuli is shown in Fig. 7. It confirms that the slope of the mass function is getting steeper with increasing distance from the cluster centre. The mass function in the annulus with distances between 0.75 and 1.5 pc, i.e., the annulus centred on the half-mass radius (see Sect. 5) provides a good fit of the overall mass function of $\mathrm{Wd} 1$. The MF slopes in the two outermost annuli (1.5 to $2.1 \mathrm{pc}$ and 2.1 to $3.4 \mathrm{pc}$, respectively) is less well defined. As discussed already in the case of the histograms, this is in part due to the relatively small number of massive stars in the outermost annulus, and in part due to uncertainties in the subtraction of field stars towards lower masses. In the following we thus assume an average slope of $\Gamma=-1.6$ for the two outermost annuli.

Assuming a single power-law initial mass function for the cluster in the mass range 0.5 to $120 M_{\odot}$ for each of the annuli, we can now estimate the total (initial) number of cluster stars. After correction for incompleteness, 1385 stars with masses between 3.4 and $30 M_{\odot}$ are present in the cluster. Below $0.5 M_{\odot}$ we assume a Kroupa IMF slope with $\Gamma=-0.3$. Table 6 lists the 

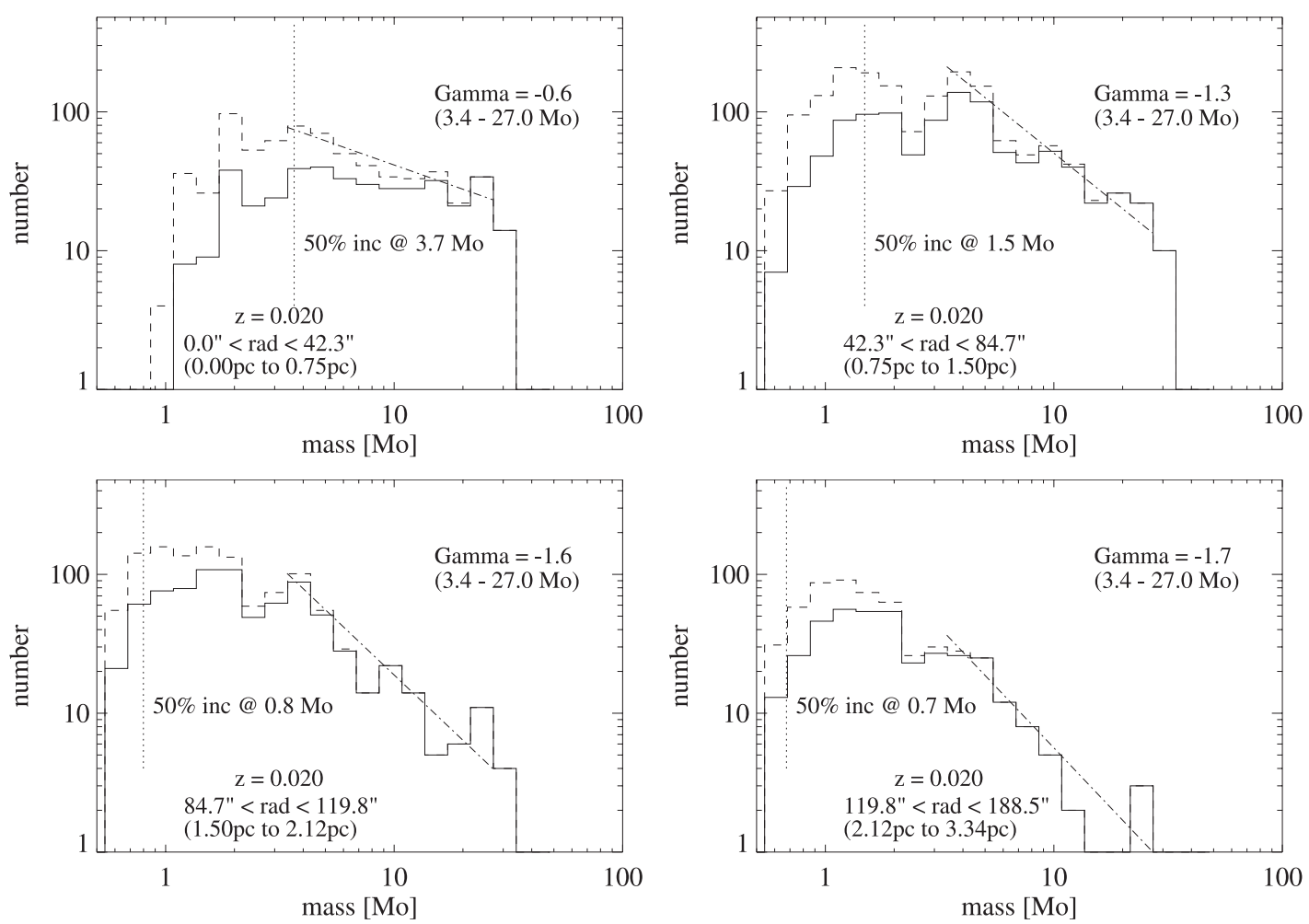

Fig. 6. Mass functions (solid line) of the cluster for 4 annuli. The dashed line gives the incompleteness corrected mass function. The $50 \%$ completeness limit is indicated by a vertical dotted line. The mass determination is based on a 3.9 Myr Geneva isochrone with $Z=0.020$ for stars with masses $\geq 6.0 M_{\odot}$, and on a 3.2 Myr Palla \& Stahler isochrone with $Z=0.019$ for stars with mass $<6.0 M_{\odot}$. The slope of the mass function (dash-dotted line) is computed from stars with masses between 3.4 and $27 M_{\odot}$.

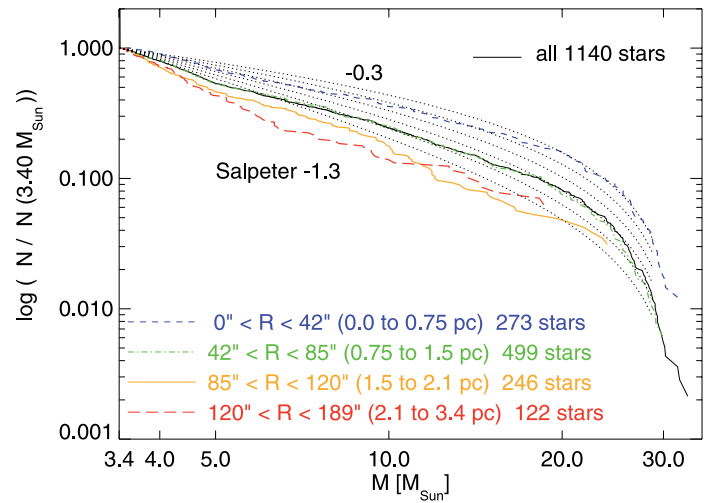

Fig. 7. Cumulative mass function for 4 annuli for stars with masses between 3.4 and $32 M_{\odot}$.

total number of stars in the cluster. Including the $\approx 65$ supernovae, which might already have exploded in $\mathrm{Wd} 1$ (see, e.g., Muno et al. 2006b, and the estimate below), the extrapolation indicates that we can expect a total of $\approx 100000$ stars with initial masses between 0.08 to $120 M_{\odot}$ in $\mathrm{Wd} 1$.

\subsection{Cluster mass}

Table 4 lists bright stars close to the saturation limit of $K \mathrm{~s}=$ $9.1 \mathrm{mag}$ in our sample, which also have spectral types determined by Clark et al. (2005). Mass estimates derived by comparison with Geneva isochrones show that these stars indeed have masses around $30 M_{\odot}$, as also determined by Clark et al. for the faint end of their sample. This overlap between the two samples enables us to study the cluster mass over the entire range from our 50\% completeness limit up to the highest mass stars identified by Clark et al. (2005).

We determine the total stellar mass of the cluster in two slightly different ways. First, we add the mass of each star detected in the SofI data, correcting this number by the completeness correction. The completeness correction was done on a starby-star basis for each star detected, taking both the brightness and the radial distance from the cluster centre into account (i.e. for a $6 M_{\odot}$ star located in the very cluster centre, the correction factor applied is higher than for a $6 M_{\odot}$ star located near the outer edge of the innermost annulus). Table 5 summarises the stellar mass in the cluster for three different annuli and stellar mass bins, only counting stars with masses between the respective $50 \%$ completeness limits and $30 M_{\odot}$. Main sequence stars with masses between $3.5 M_{\odot}$ and $30 M_{\odot}$ amount to $\approx 11300 M_{\odot}$. Including all low-mass stars with masses down to $0.8 M_{\odot}$, the total stellar mass in this mass range is at least $\approx 14700 M_{\odot}$. If we add to this the estimate from Clark et al. (2005) that stars more massive than $30 M_{\odot}$ contribute another $6000 M_{\odot}$, the combined stellar mass of Westerlund 1 amounts to at least $2 \times 10^{4} M_{\odot}$ (right column). Higher resolution adaptive optics data, which we are going to discuss in a forthcoming paper, reveal that the cluster mass function extends well below $0.8 M_{\odot}$, adding even more stars and mass to the cluster.

The second way to estimate the total stellar mass is based on the mass function slopes, and the observed number of stars with masses between 3.4 and $30 M_{\odot}$, taking the completeness correction into account. By assuming a single power law mass function in each of the cluster annuli for stellar masses between $0.5 M_{\odot}$ (the peak of the Kroupa IMF) and $120 M_{\odot}$, and assuming $\Gamma=-0.3$ for masses between 0.08 and $0.5 M_{\odot}$, we can extrapolate the total number of stars and the total stellar mass. For 
Table 4. Stars in the overlap between our sample based on NIR photometry and the optical spectroscopic studies by Clark et al. (2005) and Crowther et al. (2006).

\begin{tabular}{|c|c|c|c|c|c|c|c|c|}
\hline $\begin{array}{c}\text { W87 } \\
\#\end{array}$ & $\overline{\mathrm{RA}(2000)}$ & $\overline{\operatorname{Dec}(2000)}$ & $\begin{array}{c}K \mathrm{~s} \\
{[\mathrm{mag}]}\end{array}$ & $\begin{array}{l}J-K \mathrm{~s} \\
{[\mathrm{mag}]}\end{array}$ & $\begin{array}{c}M_{3.2 \mathrm{Myr}} \\
{\left[M_{\odot}\right]}\end{array}$ & $\begin{array}{c}M_{3.9 \mathrm{Myr}} \\
{\left[M_{\odot}\right]}\end{array}$ & $\begin{array}{c}M_{4.9 \mathrm{Myr}} \\
{\left[M_{\odot}\right]}\end{array}$ & $\overline{\mathrm{Spt}}$ \\
\hline 60 & 16474.12 & -455152.3 & $9.13 \pm 0.01$ & $1.59 \pm 0.01$ & 36.7 & 32.0 & 28.0 & O9.5Ia-B0.5Ia ${ }^{a}$ \\
\hline 29 & 16474.41 & -455040.0 & $9.17 \pm 0.03$ & $1.58 \pm 0.03$ & 36.4 & 31.7 & 27.8 & O9.5Ia-B0.5Ia \\
\hline & 16476.54 & -455039.2 & $9.25 \pm 0.05$ & $2.03 \pm 0.05$ & & & & WR77s: WN6o \\
\hline 15 & 16476.63 & -455029.8 & $9.66 \pm 0.01$ & $1.56 \pm 0.01$ & 30.6 & 27.6 & 24.8 & OB binary/blend \\
\hline & 16477.65 & -455236.0 & $9.33 \pm 0.02$ & $1.90 \pm 0.02$ & & & & WR77sb(O): WN6o ${ }^{a}$ \\
\hline
\end{tabular}

${ }^{a}$ Identified as a variable star (Bonanos 2007).

The 1st column lists the identification according to Westerlund (1987). Mass estimates (Cols. 7-9) are based on Geneva isochrones for ages of 3.2, 3.9 and 4.9 Myr, respectively. Spectral types from Clark et al. (OB-stars) and Crowther et al. (W-R stars) are listed in Col. 10.

Table 5. Integrated stellar mass for different annuli and stellar mass bins, and resulting total cluster mass including all stars detected on the SOFI frame with individual mass estimates up to $30 M_{\odot}$.

\begin{tabular}{ccccc}
\hline \hline Radial Dist. & $0.8-1.5 M_{\odot}$ & $1.5-3.5 M_{\odot}$ & $3.5-30 M_{\odot}$ & $M_{\text {tot }}$ \\
pc & {$\left[M_{\odot}\right]$} & {$\left[M_{\odot}\right]$} & {$\left[M_{\odot}\right]$} & {$\left[M_{\odot}\right]$} \\
\hline $0-0.75$ & $?$ & $>210$ & 4135 & $>4345$ \\
$0.75-1.5$ & $>220$ & 1090 & 4930 & $>6240$ \\
$1.5-2.1$ & 455 & 780 & 1825 & 3060 \\
$2.1-3.3$ & 260 & 340 & 540 & 1140 \\
\hline total mass & $>935$ & $>2420$ & 11430 & $>14785$ \\
\hline total mass $^{a}$ & & & & $>20800$ \\
$\left(0.8-120 M_{\odot}\right)$ & & & & \\
\hline
\end{tabular}

${ }^{a}$ Including the estimate of $\approx 6000 M_{\odot}$ in stellar mass for stars more massive than $30 M_{\odot}$ (Clark et al. 2005).

Mass bins below the $50 \%$ completeness limit were not corrected for incompleteness. As a lower limit, only the total mass of stars actually detected in these bins is quoted, preceded by a ">". As the field of view is limited to an area of $4.3 \mathrm{pc} \times 4.3 \mathrm{pc}$, approximately centred on the cluster, only stars located within $3.3 \mathrm{pc}$ of the cluster centre are considered.

the extrapolations we carried out Monte Carlo simulations assuming a randomly populated IMF. The simulations yield a total number of stars of $\approx 100000$ (Table 6), and a total initial stellar mass of $\approx 52000 M_{\odot}$ (Table 7) for the cluster, with an uncertainty of about $\pm 15 \%$. The Monte Carlo simulations also indicate that an uncertainty in the mass function slope in the outermost annulus by \pm 0.1 results in a change of the total cluster mass by $\pm 5 \%$. An additional uncertainty derives from the unknown binary properties of the cluster members. Both multiplicity fraction and distribution of mass ratios need to be known in order to correct any derived mass function and the total cluster mass for binary properties (see, e.g., the discussion in Stolte et al. 2005, 2006). Our seeing limited Sofi data with a resolution of $0.8^{\prime \prime}$ (i.e. $\approx 2800 \mathrm{AU}$ at the distance of $\mathrm{Wd} 1$ ) do not provide strong constraints on the binary properties, so that we currently cannot quantify the effect of unresolved binaries.

For comparison, C05 estimated a total mass of $100000 M_{\odot}$ by assuming that Wd 1 currently houses 140 stars with initial masses $\geq 30 M_{\odot}$, and then extrapolating down to stars with masses $0.08 M_{\odot}$ based on a two-part Kroupa (2002) IMF with (in our notation) $\Gamma=-1.3$ for stars with masses $\geq 0.5 M_{\odot}$, and $\Gamma=-0.3$ for stars less massive. Mengel \& Tacconi-Garman (2007) derive a dynamical mass of $6.3_{-3.7}^{+5.3} \times 10^{4} M_{\odot}$ from the velocity dispersion of four red supergiants in $\mathrm{Wd} 1$. Our estimate of a total initial cluster mass of $52000 M_{\odot}$ within $\approx 2.5 \mathrm{pc}$ of the cluster centre is in agreement with these studies. As there seem to be cluster members beyond $2.5 \mathrm{pc}$ from the cluster centre, the actual cluster mass might be even higher. This confirms that $\mathrm{Wd} 1$ is indeed one of the most massive starburst clusters in the Milky Way, and one of the best local SSC analogues.

The extrapolation based on the observed slope and the single power law mass function also indicates that the cluster initially had $\approx 65$ stars with masses between 50 and $120 M_{\odot}$. For a cluster age of $\approx 4 \mathrm{Myr}$, these stars should already have turned into supernova. As discussed by Muno et al. (2006b), this corresponds to a supernova rate of one every $\approx 15000 \mathrm{yr}$. The main effect of the supernovae on the cluster would have been the removal of any remnant gas tracing back to the formation of the cluster as well as a loss of $\approx 4700 M_{\odot}$ in stellar mass (corresponding to $\approx 10 \%$ of the initial stellar mass in the cluster). According to the IMF extrapolations, stars with masses between 30 and $50 M_{\odot}$ should have initially contributed $\approx 2400 M_{\odot}$ to the cluster mass. These stars, many of which are by now W-R stars, should have lost a total of 1000 to $1500 M_{\odot}$. Thus at the high-mass end, the cluster should have lost a total of 5700 to $6200 M_{\odot}$ in stellar mass since its formation, resulting in a current cluster mass of $\approx 45000 M_{\odot}$. Nevertheless, additional thus far undetected cluster members might be located outside the field of view of the SofI data. Thus the actual mass of $\mathrm{Wd} 1$ could be even higher.

\subsection{Effect of age uncertainties and metallicity on mass function slope and cluster mass}

As discussed before, the uncertainty in the age determination of Wd 1 traces back to the fact that we cannot a priori assume the Geneva and Palla \& Stahler isochrones to be on the same absolute timescale. Based on the width and shape of the premain sequence/main-sequence transition region, the best fitting PS99 isochrone can be determined with a small uncertainty in age. For stars with masses above $6 M_{\odot}$, the selection of the best fitting Geneva isochrone is not as straight forward.

Crowther et al. (2006) discuss that Wd 1 might have higher than solar metallicity as it is located at smaller galactocentric distances than the Sun and formed just recently. Based on the analysis of the integrated spectrum of $\mathrm{Wd} 1$, however, Piatti et al. (1998) conclude that it has close to solar metallicity. In order to get an estimate on the effect of metallicity on the derived mass function slope, we also applied a Geneva isochrone for an age of 3.9 Myr and twice solar metallicity to the observations.

Table 4 exemplifies that the selection of different Geneva isochrones with ages between $\approx 3$ and $5 \mathrm{Myr}$ and solar metallicities as well as the selection of a twice solar metallicity isochrone for an age of 3.9 Myr results in a variation of the mass estimate by $\pm 15 \%$ for the most massive stars in our sample. Towards lower masses this effect becomes less pronounced, and only the upper most bins of the mass function are affected. The estimate of the integrated cluster mass is even less subject to the uncertainties in the age estimate. Table 3 summarises the different 
Table 6. Total number of stars in the cluster based on extrapolation of the mass function slope, and scaling according to the observed number of stars with masses between 3.4 and $30 M_{\odot}$ for three annuli.

\begin{tabular}{|c|c|c|c|c|c|c|c|c|}
\hline $\begin{array}{l}\text { Radial Dist. } \\
\text { pc }\end{array}$ & $\overline{\overline{\Gamma_{\mathrm{obs}}}}$ & $\begin{array}{c}N_{\text {obs }} \\
\left(3.4-30 M_{\odot}\right)\end{array}$ & $\begin{array}{c}N_{\text {compl }} \\
\left(3.4-30 M_{\odot}\right)\end{array}$ & $\begin{array}{c}N_{0.08-0.5} \\
\left(0.08-0.5 M_{\odot}\right)\end{array}$ & $\begin{array}{c}N_{0.5-30} \\
\left(0.5-30 M_{\odot}\right)\end{array}$ & $\begin{array}{c}N_{30-50} \\
\left(30-50 M_{\odot}\right)\end{array}$ & $\begin{array}{c}N_{50-120} \\
\left(50-120 M_{\odot}\right)\end{array}$ & $\begin{array}{c}N_{\text {tot }} \\
\left(0.08-120 M_{\odot}\right)\end{array}$ \\
\hline $0-0.75$ & -0.6 & 285 & 404 & 14800 & 1585 & 37 & 42 & 15460 \\
\hline $0.75-1.5$ & -1.3 & 512 & 634 & 32700 & 8180 & 18 & 15 & 40910 \\
\hline $1.5-3.5$ & -1.6 & 322 & 347 & 36700 & 7770 & 6 & 6 & 44480 \\
\hline sum & & 1119 & 1385 & 84200 & 17530 & 61 & 63 & 101850 \\
\hline
\end{tabular}

For the estimate in each annulus, a power-law IMF with the observed $\Gamma$ is assumed over the mass range from 0.5 to $120 M_{\odot}$, and $\Gamma=-0.3$ for masses between 0.08 and $0.5 M_{\odot}$.

Table 7. Current and total initial stellar mass of the cluster based on extrapolation of the mass function slope, and scaling according to the observed number of stars with masses between 3.4 and $30 M_{\odot}$ for three annuli.

\begin{tabular}{ccccccc}
\hline \hline Radial Dist. & $\Gamma_{\text {obs }}$ & $M_{0.08-0.5 M o}$ & $M_{0.5-30 M o}$ & $M i_{30-50 M o}$ & $M_{50-120 M o}$ & $M_{\text {tot }}$ \\
pc & & {$\left[M_{\odot}\right]$} & {$\left[M_{\odot}\right]$} & {$\left[M_{\odot}\right]$} & {$\left[M_{\odot}\right]$} & {$\left[M_{\odot}\right]$} \\
\hline $0-0.75$ & -0.6 & 3110 & 5460 & 1430 & 3210 & 13210 \\
$0.75-1.5$ & -1.3 & 6940 & 12550 & 690 & 1080 & 21260 \\
$1.5-3.5$ & -1.6 & 7750 & 9400 & 275 & 430 & 17550 \\
\hline sum & & 17800 & 27410 & 2395 & 4720 & 52320 \\
\hline
\end{tabular}

For the estimate in each annulus, a power-law IMF with the observed $\Gamma$ is assumed over the mass range from 0.5 to $120 M_{\odot}$, and $\Gamma=-0.3$ for masses between 0.08 and $0.5 M_{\odot}$.

mass function slopes $\Gamma$ derived by applying Geneva isochrones with ages of 3.2, 3.9, and $4.9 \mathrm{Myr}$, respectively, to the data.

From this we can conclude that the determination of the slope of the mass function as well as the cluster mass are quite robust, and relatively insensitive to the remaining uncertainties in the age and metallicity of $\mathrm{Wd} 1$.

\section{Structural and dynamical properties of Wd 1}

\subsection{Cluster size and shape}

The identification of several 1000 cluster members with masses between 0.8 and $32 M_{\odot}$ facilitates a study of the structural and dynamical properties of the cluster.

From the distance of $3.55 \mathrm{kpc}$ between the Sun and $\mathrm{Wd} \mathrm{1,} \mathrm{a}$ distance of $8.0 \mathrm{kpc}$ between the Sun and the GC, and the Galactic coordinates of $\mathrm{Wd} 1$, we compute its galactocentric distance to $4.8 \mathrm{kpc}$. As already pointed out in the introduction, $\mathrm{Wd} 1$ is thus at a large enough distance from the GC that tidal effects leading to stripping of stars can be neglected.

Only counting stars with masses between 3.4 and $32 M_{\odot}$, the stellar surface density in the cluster core is at least $3.6 \times$ $10^{3} M_{\odot} / \mathrm{pc}^{2}$. The surface density profile follows a $\propto\left(1+(r / a)^{2}\right)^{-2}$ law with $a=1.1 \mathrm{pc}$ (see Fig. 8, left). According to Elson et al. (1987, Eq. (22)) this corresponds to a core radius of 0.4 pc. We do not attempt to fit a King profile, as the crowding and saturation of the brightest stars near the cluster centre prevents any accurate determination of the cluster's core radius based on the SofI data.

The half mass radius for all stars with masses in the range 3.4 to $32 M_{\odot}$, averaged over all position angles, is $1.0 \mathrm{pc}$ (Fig. 8, right). Figure 9 gives evidence that the cluster is not spherical. Here we compute the half mass radius as a function of position angle, taking only stars located either in a quadrant $\pm 45^{\circ}$ relative to the position angle (PA) or located in the opposite quadrant $\left(P A \pm 180^{\circ}\right)$ into account. The mass range of 3.0 to $32 M_{\odot}$ has been divided into two equal size bins in log mass. In both mass bins, the cluster is clearly elongated with its major axis along $P A \approx-10^{\circ}$ to $10^{\circ}$ and its minor axis along $P A \approx 80^{\circ}$ to $90^{\circ}$.
The elongation is more pronounced for stars with masses in the range 10 to $32 M_{\odot}$ with an eccentricity of the cluster $e=1-$ $\frac{\text { minoraxis }}{\text { majoraxis }}=0.19$ (Fig. 9, left). The average half-mass-radius for stars in this mass bin is $0.8 \mathrm{pc}$, which is in perfect agreement with the half-light radius reported by Mengel \& Tacconi-Garman (2007) based on another set of NTT/SofI observations of Wd 1. For stars with masses in the range 3.5 to $10 M_{\odot}$ the eccentricity of the cluster is $e=0.15$ and the average half mass radius is $1.1 \mathrm{pc}$ (Fig. 9, right). The larger half mass radius in the lower mass bin indicates the presence of mass segregation.

An elongation was also reported by Muno et al. (2006b) in their analysis of the shape of the diffuse X-ray emission from Wd 1. The diffuse X-ray emission shows an ellipticity of 0.75 (corresponding to an eccentricity of 0.25 ) with a major axis along $P A=13^{\circ} \pm 3^{\circ}$ and a characteristic radius (half-width at half-maximum) of $25^{\prime \prime}$ (corresponding to $0.44 \mathrm{pc}$ for a distance of $3.55 \mathrm{kpc}$ ). Muno et al. (2006b) discuss possible origins of the diffuse X-ray component. While the emission in the core of $\mathrm{Wd} 1$ can be attributed to colliding winds of $\mathrm{O}$ and W-R stars with initial masses $>15 M_{\odot}$, less than $30 \%$ of the extended diffuse emission can be explained by a spatially dispersed pre-main sequence star population with masses $\leq 2 M_{\odot}$. A possible explanation for $\approx 70 \%$ of the extended component of the diffuse emission are nonthermal particles accelerated by colliding winds and supernova remnants.

The fact that both the amount of ellipticity and the direction of the major axis of the diffuse X-ray emission are in good agreement with the structural parameters derived from the NIR data for stars with masses between 10 and $30 M_{\odot}$ supports the idea that colliding winds from the most massive stars give rise to the diffuse X-ray emission in the core of $\mathrm{Wd} 1$.

In their study of the structure and dynamics of the ONC, Hillenbrand \& Hartmann (1998) find an eccentricity of 0.30 for ONC. They conclude that rotational flattening of ONC seems unlikely and that the elongation might reflect the shape of the parental molecular cloud.

Given the still young age of $\mathrm{Wd} 1$ of 3 to $5 \mathrm{Myr}$, its elongation, too, should trace back to the formation of the cluster. Possible explanations are formation out of an elongated parental 

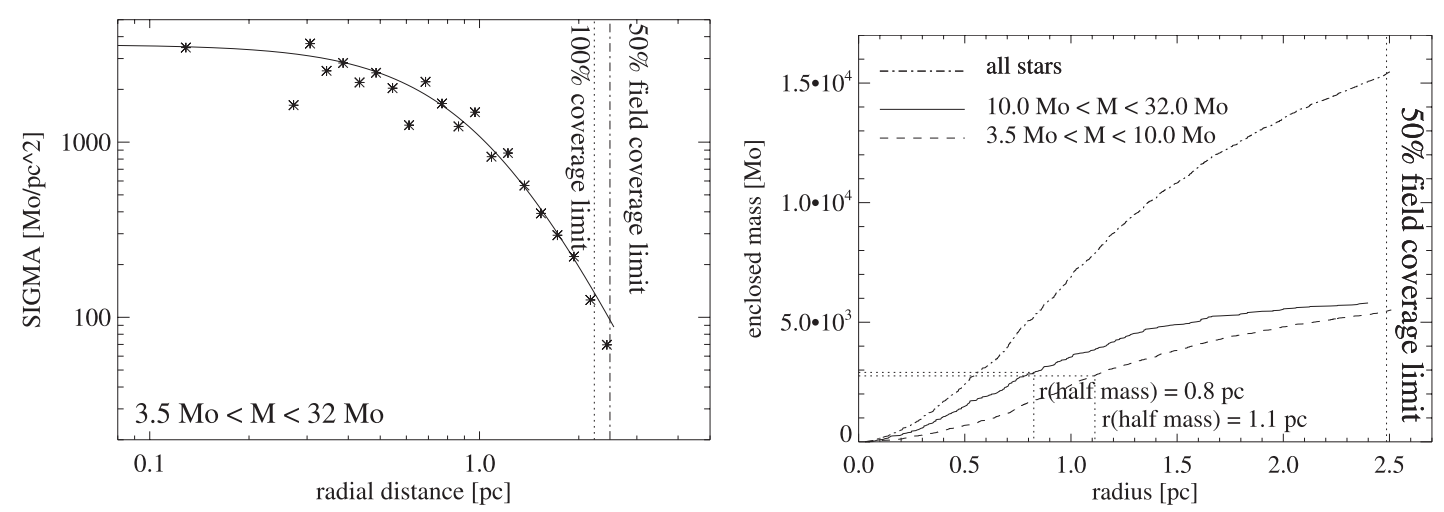

Fig. 8. Left: radial surface mass density for stars with masses between 3.5 and $32 M_{\odot}$. The central surface density is $\geq 3.6 \times 10^{3} M_{\odot} / \mathrm{pc}^{2}$. Overplotted is a function $\propto\left(1+(r / a)^{2}\right)^{-2}$ with $a=1.1 \mathrm{pc}$. Right: enclosed stellar mass as a function of radial distance from the cluster centre. Mass segregation is evident by the increasing half-mass radius with decreasing stellar mass. For stars with masses between 10 and $32 M_{\odot}$ the half-mass radius is $0.8 \mathrm{pc}$, whereas for stars with masses between 3.5 and $10 M_{\odot}$ the half-mass radius is $1.1 \mathrm{pc}$.
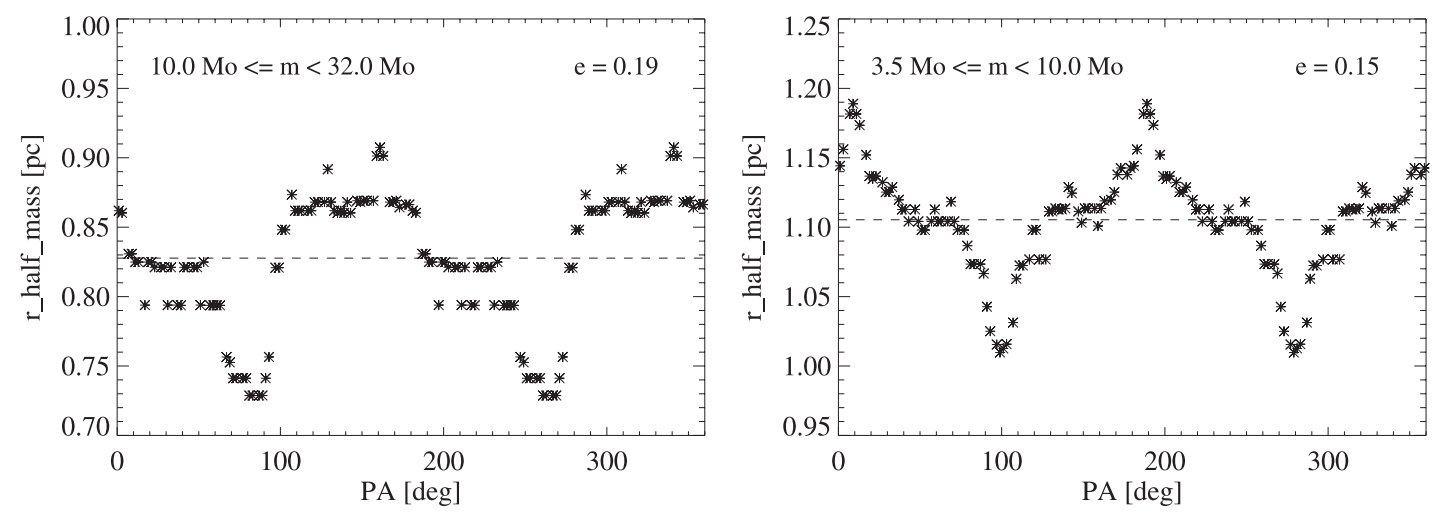

Fig. 9. The half mass radius as a function of position angle for stars with masses in the range 10 to $32 M_{\odot}$ (left) and masses in the range 3.5 to $10 M_{\odot}$ (right). The dashed line indicates the half mass radius averaged over all position angles. For both mass bins the cluster is clearly elongated, with a minor axis at $P A \approx 85^{\circ}$ and an eccentricity $=0.19$ in the high mass bin, and a minor axis at $P A \approx 100^{\circ}$ and an eccentricity of 0.15 in the low mass bin. The larger half-mass radius in the low-mass bin compared to the high mass bin indicates the presence of mass segregation. Note that we assumed the cluster half-mass radius to be point-symmetric with respect to the cluster centre.

Table 8. The decreasing half-mass radius with increasing stellar mass gives evidence for the presence of mass segregation in $\mathrm{Wd} 1$. The value in the mass bin $30-40 M_{\odot}$ is from C05.

\begin{tabular}{c|ccc}
\hline \hline Mass bin & $3-10 M_{\odot}$ & $10-30 M_{\odot}$ & $30-40 M_{\odot}$ \\
\hline$r_{\mathrm{hm}}$ & $1.1 \mathrm{pc}$ & $0.8 \mathrm{pc}$ & $0.44 \mathrm{pc}\left(25^{\prime \prime}\right)$ \\
\hline
\end{tabular}

molecular cloud, formation of the cluster out of two or multiple molecular sub-cores, out of a rotating molecular cloud, or a combination of these.

\subsection{Cluster dynamics}

According to Binney \& Tremaine (1987), the velocity dispersion $\sigma$ of a relaxed stellar system in virial equilibrium is

$\sigma=\sqrt{0.4 \times G \times M_{\mathrm{cl}} / r_{\mathrm{hm}}}$.

For a cluster mass of $\geq 45000 M_{\odot}$ and a 2-D half-mass radius of $r_{\mathrm{hm}}=1.0 \mathrm{pc}$ (see Fig. 8, right), we derive a $1-\mathrm{D}$ velocity dispersion $\sigma \geq 4.5 \mathrm{~km} \mathrm{~s}^{-1}$. This is in good agreement with the velocity dispersion of $5.0 \pm 1.7 \mathrm{~km} \mathrm{~s}^{-1}$ derived by Mengel $\&$ Tacconi-Garman (2007) based on a weighted average for radial velocity measurements of four red supergiants in $\mathrm{Wd} 1$. In terms of proper motion, $4.5 \mathrm{~km} \mathrm{~s}^{-1}$ corresponds to $0.25 \mathrm{mas} / \mathrm{yr}$ at a distance of $3.55 \mathrm{kpc}$. As shown by Stolte et al. (2008) for the Arches cluster, multi-epoch adaptive optics observations at 8 to $10 \mathrm{~m}$ class telescopes now achieve measurement accuracies better than $0.5 \mathrm{mas} / \mathrm{yr}$. Thus it should be possible to measure the velocity dispersion of a significant number of cluster stars using two epochs of high-angular resolution observations separated by a few years. From this, any deviation from virial equilibrium due to the cluster's young age can be investigated. Rotation of the cluster as a whole might result in an anisotropy in the observed velocity dispersion of stars. While in the direction parallel to the rotation axis stars should just exhibit the internal velocity dispersion of the cluster, in the direction perpendicular to the rotation axis stars should on average show a larger velocity.

The crossing time for a star located at the half-mass radius and with a velocity equal to the velocity dispersion is $t_{\text {cross }}=$ $r_{\mathrm{hm}} / \sigma=3 \times 10^{5} \mathrm{yr}$. For a cluster age of $3.6 \mathrm{Myr}$, this corresponds to $\approx 12$ crossings since the formation of the cluster, indicating that the cluster already has experienced significant dynamical relaxation and mass segregation.

Assuming a total number of cluster members $N_{\text {tot }}=10^{5}$ (Table 6), we can also compute the half-mass relaxation time (see, e.g., Spitzer \& Hart 1971):

$t_{\text {relax }}=\frac{0.2 N_{\text {tot }}}{\ln \left(0.1 N_{\text {tot }}\right)} \times \sqrt{0.4} \times t_{\text {cross }}=4 \times 10^{8} \mathrm{yr}$.

Thus even if the gaseous and stellar mass of the cluster would have been constant since its formation, it would not yet be 
Table 9. Comparison of Westerlund 1's astrophysical parameters with other young, massive clusters.

\begin{tabular}{ccccccc}
\hline \hline & $\mathrm{Wd} 1$ & $\mathrm{ONC}^{a}$ & $\mathrm{Wd}^{b}$ & NGC 3603YC $^{c}$ & Arches $^{d}$ & R136 cluster $^{e}$ \\
\hline Age [Myr] & $3.6 \pm 0.7$ & $0.3-1.0$ & $1-3$ & $1-3$ & $2-3$ & $2-3$ \\
$t_{\text {relax }}[\mathrm{Myr}]$ & 400 & 2.1 & & & $2 ?$ & \\
$r_{\text {core }}[\mathrm{pc}]$ & 0.4 & $0.16-0.21$ & $0.002-0.2$ & $?$ & $0.034-0.30$ & \\
$r_{\text {hm }}[\mathrm{pc}]$ & 1.0 & 0.8 & & $0.002-0.4$ & 0.24 & 1.1 \\
$e$ & $0.15-0.20$ & 0.30 & $?$ & $?$ & $?$ & $?$ \\
$N_{\text {tot }}$ & $10^{5}$ & $>2800$ & & $>1680$ & $>3450$ & \\
$M_{\text {tot }}\left[M_{\odot}\right]$ & $5 \times 10^{4}$ & $0.18 \times 10^{4}$ & $0.7 \times 10^{4}$ & $>0.7 \times 10^{4}$ & $>1.3 \times 10^{4}$ & $10 \times 10^{4}$ \\
(mass range in $\left.M_{\odot}\right)$ & $0.4-120$ & $0.1-35$ & $0.08-120$ & $0.1-120$ & $1-120$ & $0.5-25$ \\
\hline
\end{tabular}

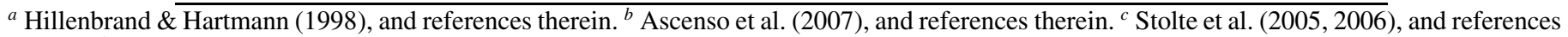
therein. ${ }^{d}$ Figer et al. (1999), Stolte et al. (2005), and references therein. ${ }^{e}$ Andersen et al. (2008), Brandl et al. (1996), and references therein.

dynamically relaxed, and further mass segregation would be expected. The expulsion of any remnant gas tracing back to the formation of the cluster by the multiple supernovae explosions, as well as the loss of $12 \%\left(\approx 6000 M_{\odot}\right)$ of the total initial stellar mass of the cluster since its formation due to stellar winds and supernova explosions keep Wd 1 out of virial equilibrium.

The effect of rotation on the dynamical evolution of a cluster was discussed by Ernst et al. (2007). They found that the presence of rotation accelerates the dynamical evolution only in systems with a single mass stellar component. In clusters with a realistic mass function, this effect disappears as mass segregation becomes the primary means to accelerate core collapse of the cluster. Thus we do not expect that any rotation of $\mathrm{Wd} 1$ should have a strong effect on its dynamical evolution.

As discussed, e.g., by Portegies Zwart et al. (2007), the presence and fraction of binary stars in a cluster can also have a strong effect on its dynamical evolution. Crowther et al. (2006) discuss evidence that at least between $1 / 3$ to $2 / 3$ of the W-R stars in Wd 1 are members of binary systems. Bonanos (2007) identifies 5 eclipsing binaries among the massive stars in Wd 1. A systematic survey for binaries and multiple systems in $\mathrm{Wd} 1$, in particular among the intermediate to low mass stars, is still missing. Thus we currently cannot quantify the binary fraction, and its effect on the dynamical evolution of $\mathrm{Wd} 1$.

\section{Comparison to the NGC 3603, Arches and 30 Doradus starburst clusters}

In Table 9 we present a compilation and comparison of astrophysical quantities of the galactic young massive clusters $\mathrm{Wd} 1$ with ONC, NGC 3603 YC, and Arches, as well as the R136 cluster in the 30 Doradus region in the Large Magellanic Cloud.

All clusters have in common that they posses at least several O-type stars with masses in excess of $30 M_{\odot}$. The large number of evolved massive stars, including hypergiants and W-R stars, present in $\mathrm{Wd} 1$ gives evidence that it is the oldest, most evolved cluster in the sample. Mass segregation seems to be a common feature among young clusters. As shown in Fig. 9, Wd 1's half-mass radius increases with decreasing stellar mass. A similar trend has been observed by Brandl et al. (1996) for the core radius of the R136 cluster. Compared to NGC 3603 YC and the Arches cluster, $\mathrm{Wd} 1$ has a rather large half-mass radius of $\approx 1.0 \mathrm{pc}$, quite similar to the half-mass radii of the ONC and the R136 cluster.

Since dynamical mass estimates have not yet been derived for any of the clusters, and since the crowding and the presence of bright stars limits the ability to detect the low-mass stellar content of these clusters, mass estimates are in general lower limits. Wd 1 with a total stellar mass between $21000 M_{\odot}$ and
$52000 M_{\odot}$ is the most massive, and with a total stellar population of up to 100000 stars also the most populous among the galactic young massive clusters presented in Table 9. Only the R136 cluster in the 30 Doradus region in the Large Magellanic Cloud, for which Andersen et al. (2008) derive a mass of 2.5 to $3 \times 10^{4} M_{\odot}$ counting stars with masses down to $2.35 M_{\odot}$, is more massive.

\section{Summary and outlook}

We have analysed near-infrared NTT/SofI observations of the starburst cluster Westerlund 1, which is among the most massive young clusters in the Milky Way. A comparison of colourmagnitude diagrams with theoretical main-sequence and premain sequence evolutionary tracks yields improved extinction, distance and age estimates of $A_{K \mathrm{~s}}=1.13 \pm 0.03 \mathrm{mag}, d=$ $3.55 \pm 0.17 \mathrm{kpc}(D M=12.75 \pm 0.10 \mathrm{mag})$ and $t=3.6 \pm 0.7 \mathrm{Myr}$, respectively. We derive the slope of the stellar mass function for stars with masses between 3.4 and $27 M_{\odot}$. In an annulus with radii between 0.75 and $1.1 \mathrm{pc}$ from the cluster centre, we get a slope of $\Gamma=-1.3$, i.e. the Salpeter slope. Closer in, the mass function of Westerlund 1 is shallower with $\Gamma=-0.6$, while at larger separations from the cluster it is getting steeper, reaching $\Gamma=-1.6$ for separations larger than $1.5 \mathrm{pc}$. This is in good agreement with the change in mass function slope found in the starburst cluster NGC 3603 YC (Stolte et al. 2006).

Only considering stars with masses between 3.0 and $32 M_{\odot}$, we derive a half-mass radius of $1.0 \mathrm{pc}$ for the cluster. $\mathrm{Wd} 1 \mathrm{ex}-$ hibits clear deviations from spherical symmetry. The distribution of stars with masses between 10 and $32 M_{\odot}$ has an eccentricity of 0.20 with the major axis aligned roughly in the North-South direction. The distribution of stars with masses between 3.0 and $10 M_{\odot}$ is elongated in the same direction with an eccentricity of 0.15 . The flattening of the cluster might be explained by rotation of the cluster along its minor axis.

By extrapolation of the observed mass function slopes for different annuli, we derive an upper limit of the total initial stellar cluster mass of $\approx 52000 M_{\odot}$. Stellar evolution at the high mass end should have reduced the total initial cluster mass by $\approx 6000 M_{\odot}$ over the past $4 \mathrm{Myr}$. By adding up the individual initial masses of stars directly detected in the cluster, we derived a lower limit for the total present cluster mass of $m \geq 2 \times 10^{4} M_{\odot}$.

With a present-day mass of 20000 to $45000 M_{\odot}$ and an initial stellar mass of $\approx 52000 M_{\odot}, \mathrm{Wd} 1$ is the most massive starburst cluster identified in the Milky Way to date, and about 10 times as massive as the ONC, and 2 to 4 times as massive a NGC 3603 YC. Additional cluster members located outside the field of view of the SofI data might push the mass of Wd 1 even higher. 
In a following paper, we will report of the analysis of highangular resolution adaptive optics data, which trace the pre-main sequence population of the cluster down to $\approx 0.2 M_{\odot}$.

Further studies should aim at determining proper motions for individual cluster members as well as more radial velocity measurements. From this precise models of the cluster kinematics and dynamics could be derived, which also might enable one to trace back the dynamical evolution of the cluster since its formation $\approx 4$ Myr ago. Wd 1, being the most massive, and one of the most nearby starburst clusters, could turn into the prime example for studies on starburst cluster formation and evolution.

Acknowledgements. We would like to thank the referee Søren Larsen for the remarks, which helped to significantly improve the paper. We are grateful to Paul Crowther, Andreas Ernst and Mike Muno for comments, which helped to improve the paper. WB acknowledges support by a Julian Schwinger fellowship of the University of California, Los Angeles. IN is supported by the Spanish Ministerio de Educación y Ciencia under grant AYA2005-00095.

\section{References}

Andersen, M., Zinnecker, H., Moneti, A., et al. 2008, ApJ, submitted Ascenso, J., Alves, J., Beletsky, Y., \& Lago, M. T. V. T. 2007, A\&A, 466, 137 Bastian, N., \& Goodwin, S. P. 2006, MNRAS, 369, L9

Bonanos, A. Z. 2007, AJ, 133, 2696

Brandl, B., Sams, B. J., Bertoldi, F., et al. 1996, ApJ, 466, 254

Brandl, B., Brandner, W., Eisenhauer, F., et al. 1999, A\&A, 352, L69

Brandner, W., Grebel, E., Chu, Y.-H., et al. 2000, AJ, 119, 292

Binney, J., \& Tremaine, S. 1987, Galactic Dynamics (Princeton Univ. Press), p. 214

Clark, J. S., \& Negueruela, I. 2002, A\&A, 396, L25

Clark, J. S., Negueruela, I., Crowther, P. A., \& Goodwin, S. P. 2005, A\&A, 434, 949

Cotera, A. S., Erickson, E. F., Colgan, S. W. J., et al. 1996, ApJ, 461, 750

Crowther, P. A., Hadfield, L. J., Clark, J. S., Negueruela, I., \& Vacca, W. D. 2006, MNRAS 372, 1407

de Grijs, R., \& Parmentier, G. 2007, ChJA\&A, 7, 155

Devillard, N. 2001, ESO C Library for an Image Processing Software Environment (eclipse), in ADASS X, ASP Conf. Ser., 238, ed. F. R. Harnden, F. A. Primini, H. E. Payne, 525

Eisenhauer, F., Genzel, R., Alexander, T., et al. 2005, ApJ, 628, 246

Elson, R.A Fall, S. M., \& Freeman, K. C. 1987, ApJ, 323, 54

Ernst, A., Glaschke, P., Fiestas, J., Just, A., \& Spurzem, R. 2007, MNRAS, 377, 465

Figer, D. F., Kim, S.S, Morris, M., et al. 1999, ApJ, 525, 750

Figer, D. F., Najarro, F., Gilmore, D., et al. 2002, ApJ, 581, 258
Figer, D. F., Rich, R. M., Kim, S. S., et al. 2004, ApJ, 601, 319 Hadfield, L. J., \& Crowther, P. A. 2006, MNRAS, 368, 1822 Hillenbrand, L. A. 1997, AJ, 113, 1733

Hillenbrand, L. A., \& Hartmann, L. W. 1998, ApJ, 492, 540

Kim, S. S., Figer, D. F., Kudritzki, R. P., \& Najarro, F. 2006, ApJ, 653, L113

Klessen, R. S., Spaans, M., \& Jappsen, A.-K. 2007, MNRAS, 374, L29

Kothes, R., \& Dougherty, S. M. 2007, A\&A, 468, 993

Kron, G. E., \& Mayall, N. U. 1960, AJ, 65, 581

Kroupa, P. 2002, Science, 295, 82

Kroupa, P., Boily, \& C. M. 2002, MNRAS, 336, 1188

Lada, C. J., \& Lada, E. A. 2003, ARA\&A, 41, 57

Lamzin, S. A. 2006, AstL, 32, 176

Larsen, S. S., \& Richtler, T. 2004, A\&A, 427, 495

Larson, R. B. 2006, RMXAA, 26, 55

Lejeune, T., \& Schaerer, D. 2001, A\&A, 366, 538

Maíz-Apellániz, J., \& Úbeda, L. 2005, ApJ, 629, 873

Mayne, N. J., Naylor, T., Littlefair, S. P., Saunders, E. S., \& Jeffries, R. D. 2007, MNRAS, 375, 1220

McCrady, N., Graham, J. R., \& Vacca, W. D. 2005, ApJ, 621, 278

Mengel, S., \& Tacconi-Garman, L. E. 2007, A\&A, 466, 151

Meynet, G., Mermilliod, J.-C., \& Maeder, A. 1993, A\&AS, 98, 477

Morris, M. 1993, ApJ, 408, 496

Muno, M. P., Clark, J. S., Crowther, P. A., et al. 2006a, ApJ, 636, L41

Muno, M. P., Law, C., Clark, J. S., et al. 2006b, ApJ, 650, 203

Negueruela, I., \& Clark, J. S. 2005, A\&A, 436, 541

Palla, F., \& Stahler, S. W. 1999, ApJ, 525, 772

Patriarchi, P., Morbidelli, L., Perinotto, M., \& Barbaro, G. 2001, A\&A, 372, 644

Piatti, A. E., Bica, E., \& Clariá, J. J. 1998, A\&AS, 127, 423

Portegies Zwart, S. F., McMillan, S. L. W., \& Makino, J. 2007, MNRAS 374, 95

Reid, M. J. 1993, ARA\&A, 31, 345

Rieke, G. H., \& Lebofsky, M. J. 1985, ApJ, 288, 618

Rieke, G. H., Loken, K., Rieke, M. J., \& Tamblyn, P. 1993, ApJ, 412, 99

Siess L., Dufour E., \& Forestini M. 2000, A\&A, 358, 593

Skinner, S. L., Simmons, A. E., Zhekov, S. A. et al. 2006a, ApJ, 639, L35

Skinner, S. L., Perna, R., \& Zhekov, S. A. 2006b, ApJ, 653, 587

Spitzer, L., \& Hart, M. H. 1971, ApJ, 164, 399

Stahler, S. W. 1983, ApJ, 274, 822

Stetson, P. B. 1987, PASP, 99, 191

Stolte, A., Grebel, E., Brandner, W., \& Figer, D. 2002, A\&A, 394, 459

Stolte, A., Brandner, W., Brandl, B., Zinnecker, H., \& Grebel, E. K. 2004, AJ, 128,765

Stolte, A., Brandner, W., Grebel, E. K., Lenzen, R., \& Lagrange, A.-M. 2005, ApJ, 628, 113

Stolte, A., Brandner, W., Brandl, B., \& Zinnecker, H. 2006, AJ, 132, 253

Stolte, A., Ghez, A. M., Morris, M., et al. 2008, ApJ, in press

Westerlund, B. E. 1961, PASP, 73, 51

Westerlund, B. E. 1987, A\&AS, 70, 311

Whitmore, B. C., \& Schweizer, F. 1995, AJ, 109, 960

Yi, S., Demarque, P., Kim, Y.-C., et al. 2001, ApJS, 136, 417 